Open Access

\title{
Magnetohydrodynamics modeling of coronal magnetic field and solar eruptions based on the photospheric magnetic field
}

Satoshi Inoue 1,2

\begin{abstract}
In this paper, we summarize current progress on using the observed magnetic fields for magnetohydrodynamics (MHD) modeling of the coronal magnetic field and of solar eruptions, including solar flares and coronal mass ejections (CMEs). Unfortunately, even with the existing state-of-the-art solar physics satellites, only the photospheric magnetic field can be measured. We first review the 3D extrapolation of the coronal magnetic fields from measurements of the photospheric field. Specifically, we focus on the nonlinear force-free field (NLFFF) approximation extrapolated from the three components of the photospheric magnetic field. On the other hand, because in the force-free approximation the NLFFF is reconstructed for equilibrium states, the onset and dynamics of solar flares and CMEs cannot be obtained from these calculations. Recently, MHD simulations using the NLFFF as an initial condition have been proposed for understanding these dynamics in a more realistic scenario. These results have begun to reveal complex dynamics, some of which have not been inferred from previous simulations of hypothetical situations, and they have also successfully reproduced some observed phenomena. Although MHD simulations play a vital role in explaining a number of observed phenomena, there still remains much to be understood. Herein, we review the results obtained by state-of-the-art MHD modeling combined with the NLFFF.
\end{abstract}

Keywords: Sun, Magnetic field, Photosphere, Corona, Magnetohydrodynamics (MHD), Solar active region, Solar flare, Coronal mass ejection (CME)

\section{Review}

\section{Introduction}

Solar flares are explosive phenomena observed in the atmosphere of the Sun (the solar corona). These events are observed as sudden bursts of electromagnetic radiation, such as extreme ultraviolet radiation (EUV), X-rays, and even white light; some examples are shown in Fig. 1a-c. The scale is classified as soft X-rays, using the 1-8 $\AA$ band obtained by the GOES- 5 satellite (one of the Geostationary Orbiting Environment Satellites), as shown in Fig. 1d. The Sun is known to be a magnetized star. Figure 2a shows the line-of-sight component of the magnetic field, and the positive and negative polarities cover the whole sun. Figure $2 b$ shows the three-dimensional (3D) magnetic

\footnotetext{
Correspondence: inoue@mps.mpg.de

${ }^{1}$ Max-Planck Institute for Solar System Research, Justus-von-Liebig-Weg 3,

37077 Göttingen, Germany

2 Institute for Space-Earth Environmental Research, Nagoya University,

Chikusa-ku, Furo-Cho, 446-701 Nagoya, Japan
}

field lines traced from the positive to the negative polarities; these have been extrapolated under the assumption of the potential field approximation (this will be discussed below). Solar flares often occur above the sunspots corresponding to a cross section of strong magnetic flux. In addition, because the solar corona satisfies the low- $\beta$ plasma condition ( $\beta=0.01-0.1)$ (Gary 2001) in which the magnetic energy dominates that of the coronal plasma, solar flares are widely considered to be a manifestation of the conversion of the magnetic energy of the solar corona into kinetic and thermal energy, culminating in the release of high-energy particles and electromagnetic radiation. Figure $2 \mathrm{c}$ is an enlarged view of the region that is marked by an arrow in Fig. 2b; here, the field lines are responsible for the current density accumulation, which initiates the flare. These field lines are extrapolated using the nonlinear force-free field (NLFFF) approximation; this is one of the main topics of this paper. 


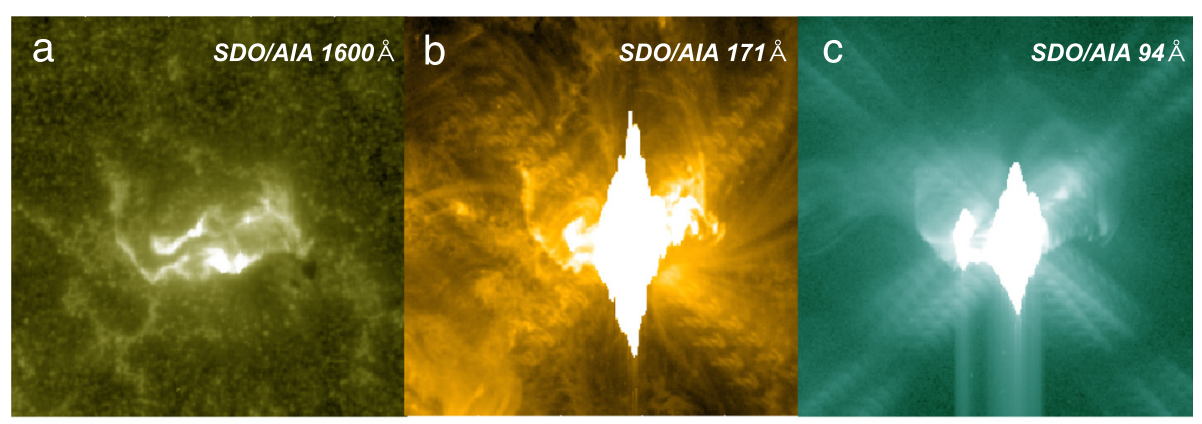

d

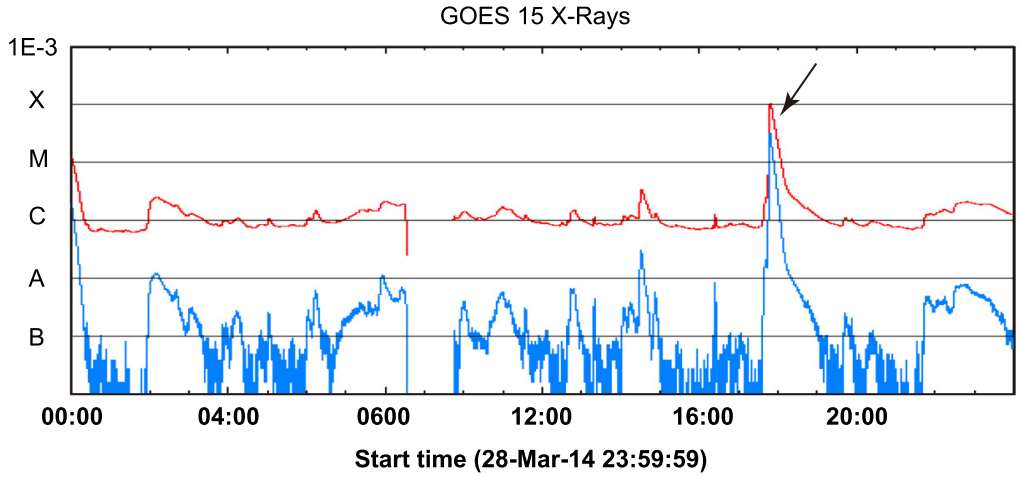

Fig. 1 Observations of the solar flare. a-c The solar flares in the EUV images for different wavelengths observed on the solar surface or in the solar atmosphere. From left to right, the wavelengths are 1600, 171, and $94 \AA$. The flares were observed by SDO/AIA at around 18:00 UT on 29 March 2014. d Time profile of the X-ray flux measured by the GOES 12 satellite on 29 March 2014. The solar X-ray outputs in the 1-8 $\AA$ and $0.5-4.0 \AA$ passbands are plotted

Furthermore, this causes a huge amount of coronal gas (a typical mass is $10^{15} \mathrm{~g}$ ) with a velocity of $100-$ $2000 \mathrm{kms}^{-1}$ to be released into interplanetary space; this is called a coronal mass ejection (CME; e.g., Forbes (2000)). The CMEs are sometimes associated with solar flares; however, the detailed understanding of the relationship between these two phenomena remains elusive (Chen 2011; Schmieder et al. 2015). It is important to understand these phenomena in order to better understand the nonlinear plasma dynamics of the processes involving the magnetic energy or helicity of the solar coronal plasma; this includes storage-and-release processes as well as the forecasting space weather (Tóth et al. 2005; Liu et al. 2008; Kataoka et al. 2014). Investigations of solar flares and CMEs are thus important in terms of both the elemental plasma physics and the applied science.

Since the discovery of the solar flares by Carrington (1859), many studies have been performed (including observational, theoretical, and numerical studies) for clarifying their dynamics (Benz 2008; Priest and Forbes 2002; Shibata and Magara 2011; Wang and Liu 2015). Many new insights on solar flares and related phenomena have been obtained by analyzing the data collected by satellites. For instance, the Yohkoh satellite obtained much data on dynamical features of the sun, some of which had not been predicted; this can be seen in Fig. 3a; this image, taken by a soft X-ray telescope, shows several important aspects that have helped our understanding of solar flares. For example, Tsuneta et al. (1992) discovered the cuspshaped structure during the solar flare seen in the lower right panel in Fig. 3a. A detailed analysis (Tsuneta 1996) produced evidence of the reconnection, and this lent support to a theoretical flare model based on reconnection; this model is named for its developers, Carmichael, Surrock, Hirayama, Kopp, and Pneumann (CSHKP) and explains the observations at multiple wavelengths (Carmichael 1964; Hirayama 1974; Kopp and Pneuman 1976; Sturrock 1966). Masuda et al. (1994) confirmed the CSHKP model of the solar flare by analyzing the hard $\mathrm{X}$-ray signals obtained during a solar flare. In addition, Sterling and Hudson (1997) found a characteristic pattern of X-rays that are released prior to a flare; this is shown in the upper right panel of Fig. 3a. This pattern is a sigmoid (an S- or inverse S-shaped structure) that changes into a cusp-shaped loop structure after the flare occurs. Su et al. (2007) and McKenzie and Canfield (2008) demonstrated the fine structure and topology of the field lines that were later observed by an X-ray telescope (Golub et al. 2007) on board the Hinode satellite (Kosugi et al. 2007). In addition, Yokoyama et al. (2001) found evidence of reconnection 

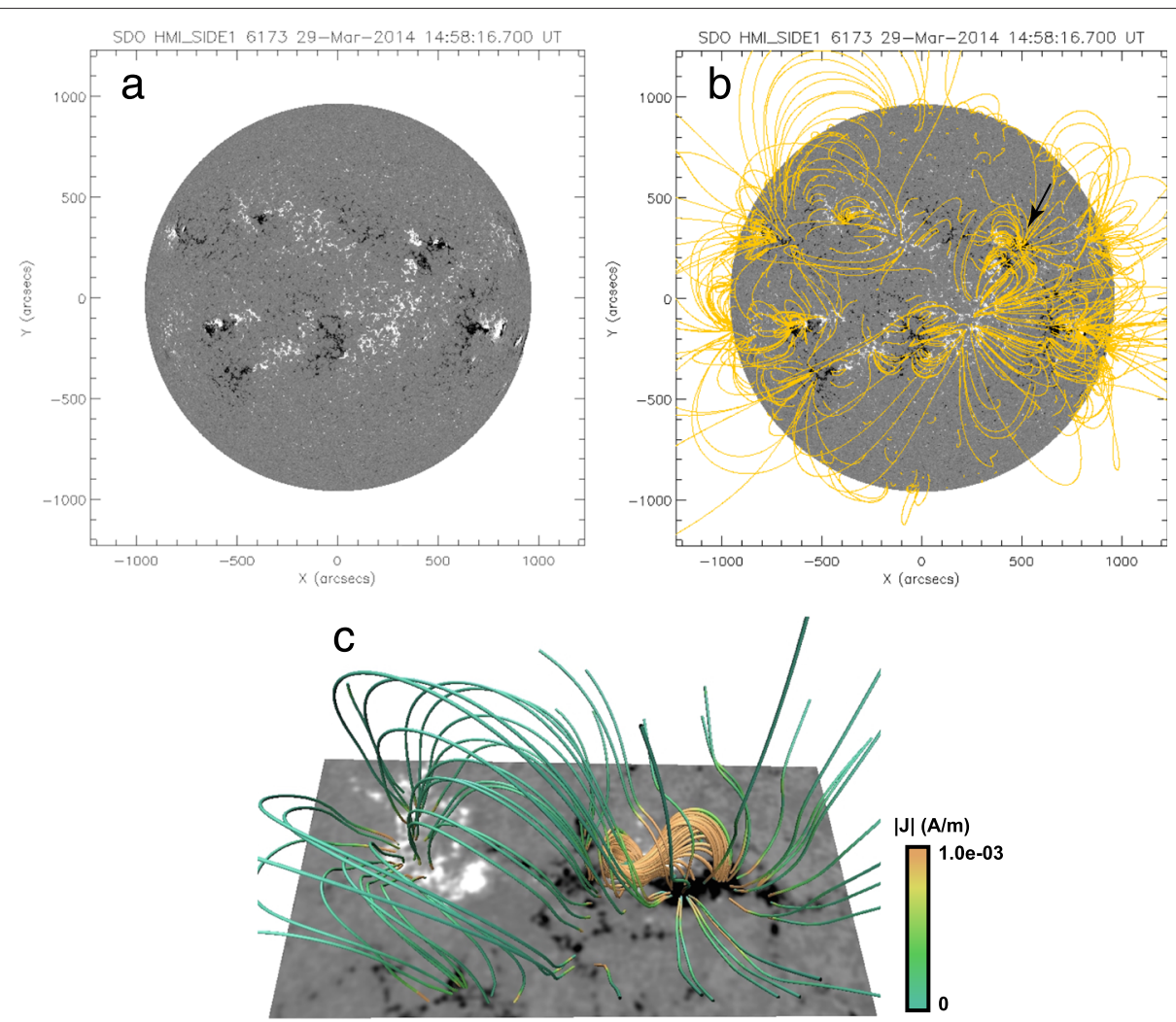

Fig. 2 Magnetic fields of the sun. a Full-disk image of a line-of-sight component of the solar magnetic field observed by SDO/HMI at 15:00 UT on 29 March 2014, which corresponds to $2.5 \mathrm{~h}$ before an X1.0-class flare. $\mathbf{b}$ The magnetic field lines in yellow are superimposed on $\mathbf{a}$. The field lines are extrapolated under the approximated potential field. This figure is courtesy of Dr. D. Shiota (Shiota et al. 2012). c The active region, corresponding to the region marked by an arrow in $\mathbf{b}$, is the region in which a sunspot with a strong magnetic field is concentrated. The field lines are plotted according to the NLFFF approximation, in which they accumulate the strong current density

inflow in extreme ultraviolet observations of the Solar and Heliospheric Observatory (SOHO). The images of the coronal loop shown in Fig. 3b are reminiscent of reconnection. Because these observations were based on imaging of electromagnetic waves, the data were mapped onto a 2D plane. Thus, obtaining a 3D reconstruction of these events is extremely difficult.

Based on this observational evidence, there have been several attempts to construct the 3D magnetic structure (e.g., Shibata (1999)). Figure 3c is an image of a 3D magnetic structure inferred from observations during the onset of the solar eruption depicted in Shiota et al. (2005); the reconnection model can be used to explain various observed phenomena, e.g., the two $\mathrm{H}$ $\alpha$ flare ribbons, and giant arcades. In addition, various models have been proposed that predict the onset of solar flares and CMEs. For instance, Forbes and Priest (1995) proposed the catastrophic model shown in Fig. 3d; this shows that the flux tube in the solar corona does not remain at equilibrium when the boundary conditions are changed, and this results in a sudden eruption. The tether-cutting model, proposed by Moore et al. (2001), is shown in Fig. 3e. They assumed that two sheared field lines existed along the polarity inversion line (PIL) prior to the onset of the flare; this is shown in the upper left panel of Fig. 3e. Note that this has a somewhat sigmoidal structure. If there is reconnection between the sheared field lines, then long twisted lines are formed, and an eruption may occur. The final state shown in the right bottom panel of Fig. 3e is very similar to that shown in Fig. 3c.

The dramatic increase in computer power allows us to perform 3D magnetohydrodynamics (MHD) simulations and to estimate the 3D dynamics of magnetic fields during solar flares. Several studies have modeled sunspots to be asymmetric or as simple dipole fields and have analytically obtained the 3D coronal magnetic fields by fitting appropriate boundary conditions (e.g., Amari et al. (2000) and Amari et al. (2003a)). Figure 4a shows the results from Amari et al. (2003b); this shows the formation of a flux tube, which is initiated by the initial potential field, through twisted and converged motion on the photosphere. The twisted motion imposed on a dipole sunspot causes the accumulation of sheared field lines, and the 
a

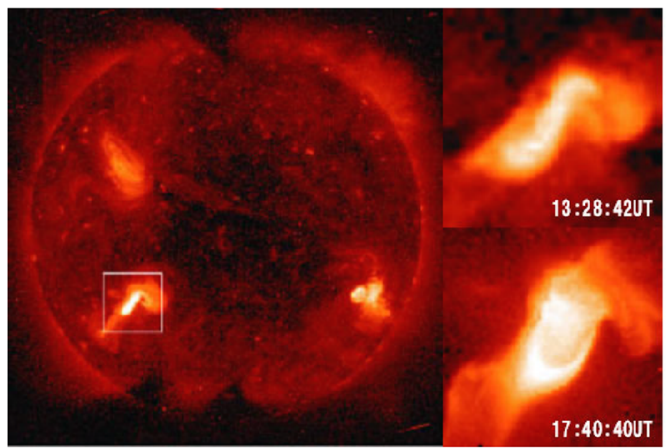

b

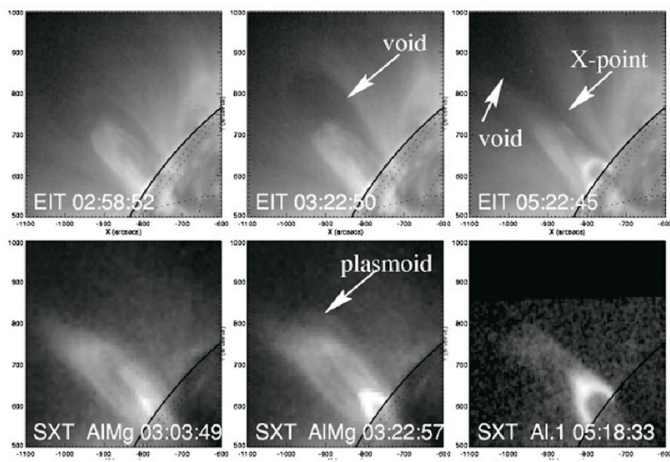

e
C

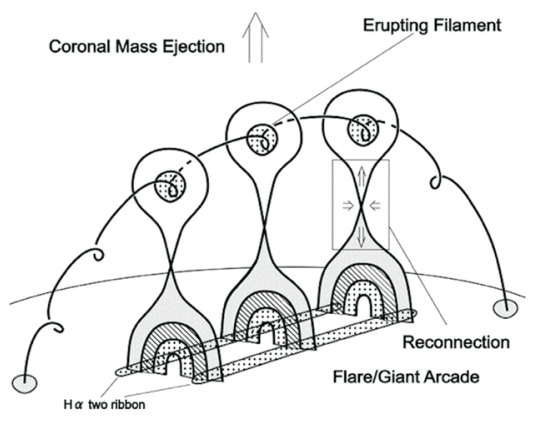

d

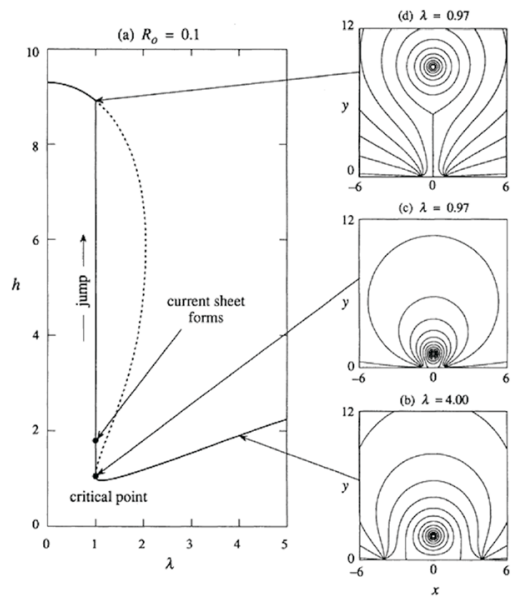

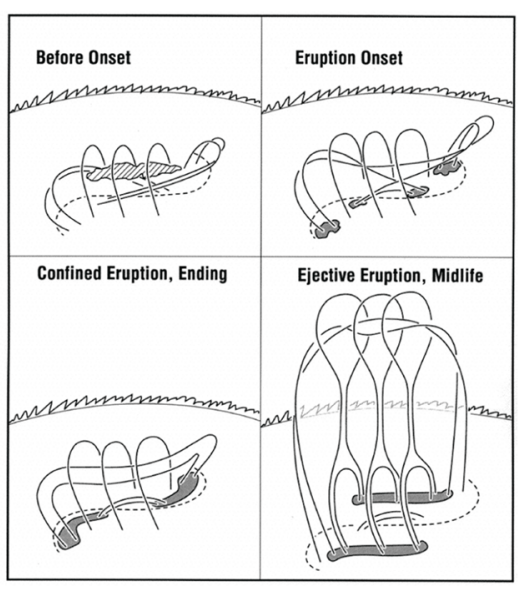

Fig. 3 Observations and models of the solar flares. a The solar corona observed by soft X-ray from on board the Yohkoh satellite. The left panel shows the whole sun; the upper and lower right panels show the sigmoid and cusp-loop structures, observed before and after the flare, respectively. This figure is courtesy of ISAS/JAXA. b The reconnection process in the solar flare observed by SOHO satellite from Yokoyama et al. (2001). c 3D view of the magnetic field during the solar flare inferred from the observations from Shiota et al. (2005). d The loss-of-equilibrium model proposed by Forbes and Priest (1995). The flux tube loses the equilibrium by changing the boundary conditions; as a result, an eruption occurs. e The tether-cutting reconnection model proposed by Moore et al. (2001). The flux tube is created by the reconnection taking place between the two sheared field lines formed before onset; eventually, the flux tube can erupt away from the solar surface. The images in (b-e) are copyright AAS and reproduced by permission

motion converging toward the PIL creates a flux tube composed of highly twisted field lines due to the flux cancellation. Aulanier et al. (2012) and Janvier et al. (2013) constructed similar MHD models, and these generated a $3 \mathrm{D}$ view that extended the well-established 2D CSHKP model. This view produced a $3 \mathrm{D}$ feature that was not seen in the $2 \mathrm{D}$ model; their simulations produced strong-toweak sheared post-flare loops, which are consistent with observations (Asai et al. 2003). On the other hand, Kusano et al. (2012) successfully reproduced an eruption in a different way, as shown in Fig. 4b. They created a linear force-free field that had shearing field lines as the initial condition; a small dipole emerging flux was imposed at a local area on the PIL. They found that only two types of emerging flux can produce a flux tube; this shows that the eruption is due to interactions with a pre-existing sheared magnetic field. Later, this scenario was confirmed in observations by Toriumi et al. (2013) and Bamba et al. (2013).

Other MHD models have been derived from an initialized flux tube. Solar filaments are often observed on the sun; these are composed of a denser plasma than that in the solar corona (Parenti 2014). It is widely agreed that the highly helical twisted lines in the filament sustain the dense plasma in the solar corona (Priest and Forbes 2002). Recent observations clearly show the helical structure of the magnetic field, i.e., the flux tube and the dynamics (e.g., Cheng et al. (2013); Nindos et al. (2015); Vemareddy and Zhang (2014)). In addition to this, the flux tube/filaments have often been observed to erupt 
a

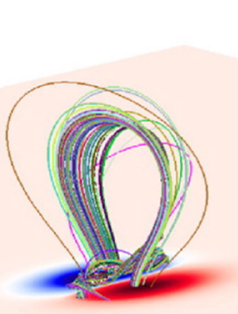

(a)

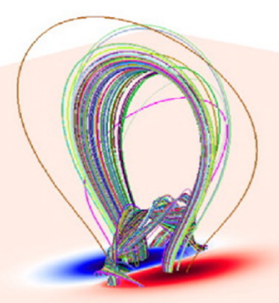

(b)

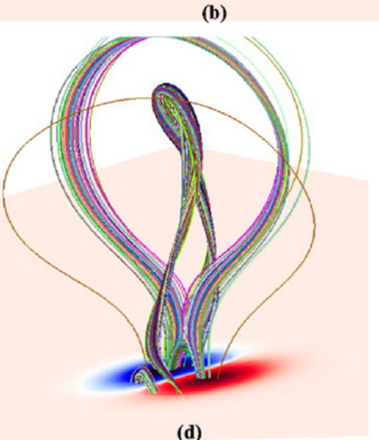

(d)

C

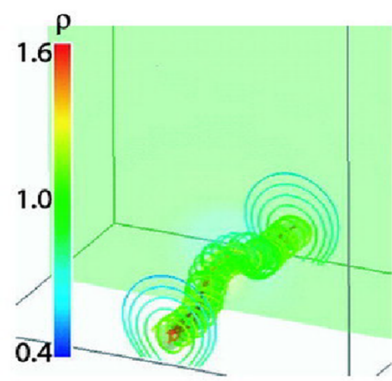

$\mathrm{T} \mid \mathrm{ME}=9.42$

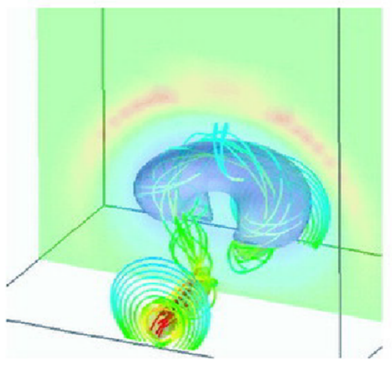

TIME $=14.80$

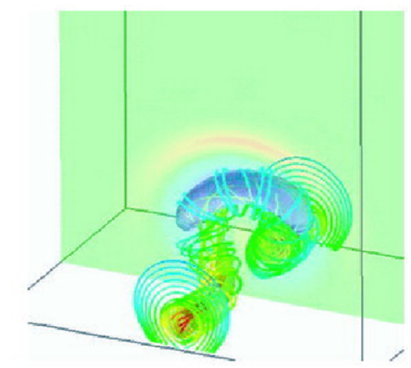

$\mathrm{T} I \mathrm{ME}=12.11$

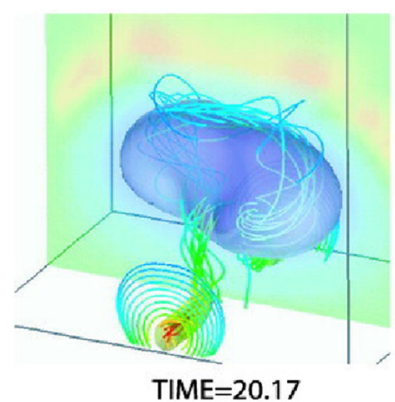

b

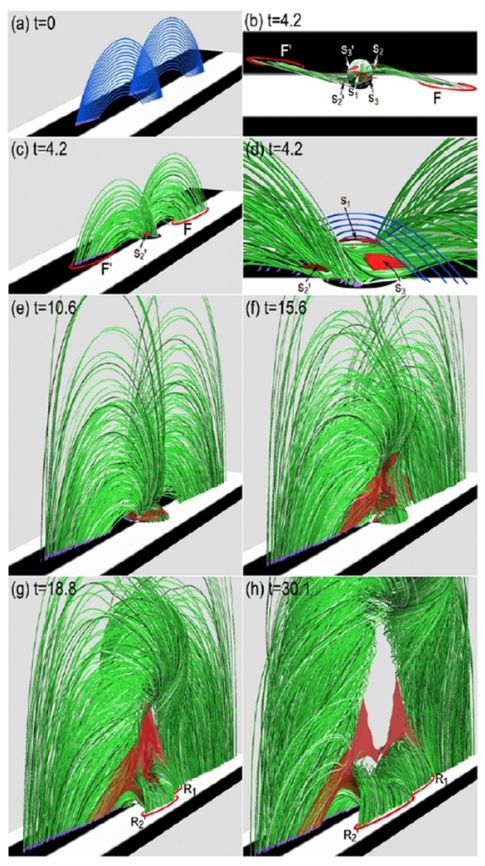

d

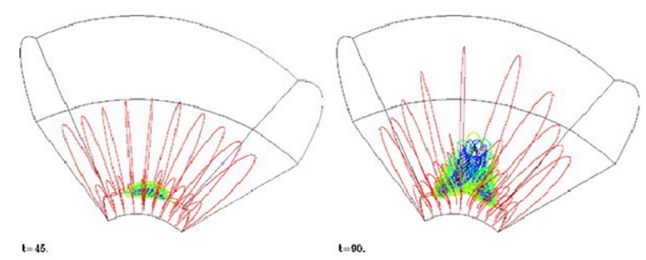

$t=48$

$t=0$

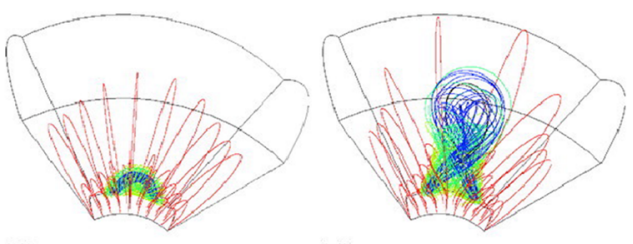

$i=x$

t=er.

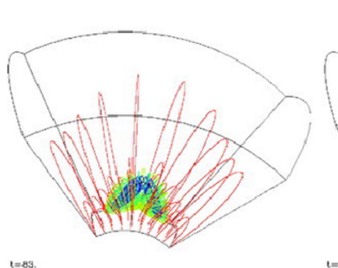

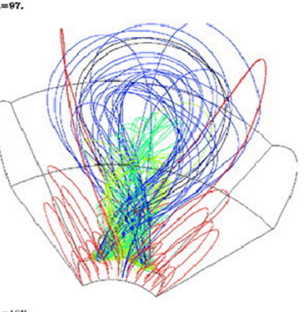

Fig. 4 3D MHD simulation of solar flares by pioneers in the field. a MHD modeling of the solar flare by Amari et al. (2003a). The potential field was reconstructed from the given simple dipole fields, which were imposed on the twisted and converged motion. Consequently, the potential field was converted into a non-potential field, leading to the eruption. b MHD modeling by Kusano et al. (2012) shows that the emergence of small flux can destroy the initial equilibrium condition of the linear force-free field, leading to the formation of a large flux tube and an eruption. $\mathbf{c}$ Inoue and Kusano (2006) investigated the flux tube dynamics associated with the solar flares and causing a CME. The flux tube was assumed to be infinitely long and was driven by kink instability, leading to a CME for a certain supra-threshold height. d Fan (2005) employed a more realistic flux tube (Titov and Démoulin 1999) with footpoints tied to the solar surface. The eruption was first driven by kink instability and later by torus instability (Fan 2010). All images are copyright AAS and reproduced by permission 
away from the solar surface. Following these observations, extensive MHD modeling, focusing on the flux tube dynamics, has been performed. Inoue and Kusano (2006) investigated the dynamics of a flux tube that was initially embedded in the solar corona, as shown in Fig. 4c. This extended the studies of Forbes (1990) and Forbes and Priest (1995) showing the dynamics in a 2D space. This study found that the flux tube eruption was caused by a kink instability in 3D space, rather than by a loss of equilibrium in 2D space, as discussed by Forbes (1990). Recently, a higher-resolution simulation was performed by Nishida et al. (2013), who reported complex reconnections and plasmoid motions associated with flux tube eruption. Chen and Shibata (2000) numerically confirmed that a flux tube eruption is triggered by a small emerging flux that is the result of the reconnection with magnetic fields lines surrounding the flux tube, and it can reduce the downward tension force acting on the flux tube. Török et al. (2009) extended this into 3D space. As shown in Fig. 4d, Török and Kliem (2005) and Fan (2005) constructed more realistic MHD models by noting that the flux tube roots are tied to the solar surface (Titov and Démoulin 1999), rather than by assuming infinitely long flux tubes as in Inoue and Kusano (2006) and Nishida et al. (2013). Török and Kliem (2005) reported that the eruption depends on the decay rate of the external magnetic field, and later, this scenario was explained as torus instability (Kliem and Török 2006). To address this instability, detailed stability and equilibrium analyses of flux tubes in the solar corona were performed by Isenberg and Forbes (2007) and Démoulin and Aulanier (2010), and the dynamics were numerically confirmed by Török and Kliem (2007), Fan (2010), and Aulanier et al. (2010). Attempts are being made to meet the challenge of simulating a solar eruption through the emergence of highly twisted flux tube embedded in the convection zone (e.g., An and Magara (2013); Archontis et al. (2014); Leake et al. (2014)).

Several studies have shown the formation and dynamics of a large-scale CME in the range of a few solar radii. Antiochos et al. (1999) proposed a breakout model in which a moving magnetic field surrounding the core fields triggers the CME; those dynamics were later confirmed in a highresolution simulation (e.g., Lynch et al. (2008) and Karpen et al. (2012)). Shiota et al. (2010) reported that an interaction between the core field (modeled as a spheromak) and the ambient field is important for determining whether an ejection will occur.

However, most of the studies presented above assumed hypothetical and ideal situations. Although these studies clarified many elementary physical processes related to the onset and dynamics of solar flares, they did not incorporate the data collected by solar satellites (in particular, they did not incorporate magnetic field data). One of the reasons for this is that only the photospheric magnetic field can be measured, and this implies that the coronal magnetic field cannot be observed directly. Nevertheless, several models have been proposed in which the photospheric magnetic field is treated as a boundary surface (.e.g., Török et al. (2011); van Driel-Gesztelyi et al. (2014); Zuccarello et al. (2012)). Challenging simulations considered a wide domain that extended from the Sun to the Earth; their major objectives included the initiation of a CME, its propagation in interplanetary space, and ultimately its interaction with the magnetosphere, which governs the dynamics of the ionosphere (Manchester et al. 2004; Tóth et al. 2005).

On the other hand, most of these models employed only the normal components of the magnetic field, neglecting the horizontal fields. Horizontal magnetic fields are very important for explaining the solar flares because these fields serve as a proxy for the extent to which the field lines are twisted and sheared, i.e., for determining the free magnetic energy at the solar surface. The MHD modeling of solar eruptions, which accounts for the three components of the photospheric magnetic field, has only recently been demonstrated, thanks to a state-of-art solar physics satellite. However, several problems remain open; these include the uniqueness of the numerical solution and the mathematical consistency of the MHD equations on a specified boundary (these questions will be discussed below).

In this paper, we present state-of-the-art MHD modeling, which accounts for the photospheric magnetic field, and we will focus on applying this to solar eruptions. In particular, we introduce the modeling of the coronal magnetic field and solar eruptions, based on the three components of the photospheric magnetic field. This area of research has been recently revived, beginning with a study by Jiang et al. (2013), and followed by Inoue et al. (2014a), Amari et al. (2014), and Inoue et al. (2015). The structure of this article is as follows. We first introduce a method for 3D reconstruction of the coronal magnetic field, based on the photospheric magnetic field; this includes a potential field that is easily reconstructed from one of the components of these fields and a nonlinear force-free field that is based on all of the components. Next, we describe recent MHD models that use a magnetic field that is reconstructed from the measured photospheric field. Finally, we draw some important conclusions.

\section{Extrapolation of the coronal magnetic fields}

Because we can obtain observations of the magnetic field components only for the photosphere, it is necessary to extrapolate to obtain information about the 3D coronal magnetic fields in $3 \mathrm{D}$. The solar corona is considered to be in low- $\beta$ plasma state, where $\beta=P / 2 B^{2}$ is defined to be the ratio of the plasma gas pressure $(P)$ to the magnetic 
pressure $\left(B^{2}\right)$. From this, we have that the force-free state

$$
\boldsymbol{J} \times \boldsymbol{B}=0
$$

is a good approximation for describing the state of the coronal magnetic field, where $\boldsymbol{B}$ is the magnetic field satisfying the solenoidal condition,

$$
\nabla \cdot B=0,
$$

and $\boldsymbol{J}$ is the current density,

$$
\boldsymbol{J}=\nabla \times \boldsymbol{B} .
$$

In this section, we introduce a method for extrapolating the solar coronal magnetic field given only the photospheric magnetic fields in the force-free approximation.

\section{Potential field}

The potential field is the simplest force-free field approximation:

$$
\nabla \times \boldsymbol{B}=0,
$$

where the current density vanishes everywhere. In this formulation, the magnetic field can be replaced with the scalar function $\psi$, as follows:

$$
\boldsymbol{B}=-\nabla \psi \text {. }
$$

If we use the solenoidal condition of Eq. (2), then Eq. (5) can be rewritten as

$$
\nabla^{2} \psi=0 .
$$

This corresponds to the Poisson equation, for which a unique solution is guaranteed for a boundary valueproblem. In this way, we can calculate the solar coronal magnetic field, given the normal component of the magnetic field $\left(B_{n}\right)$ and its Neumann condition,

$$
B_{n}=\frac{\partial \psi}{\partial n}
$$

on each boundary. Although the photospheric magnetic field can be considered to be the bottom surface, conditions are required on the other boundaries in order to solve Eq. (6). Several such methods have been proposed, some of which are described below.

One approach is to use Green's functions (Sakurai 1982; 1989). In this approach, the potential field is created by monopoles that are located at different points on the bottom boundary $\left(x^{\prime}, y^{\prime}, 0\right)$, at which the magnetic flux $B_{z} d x^{\prime} d y^{\prime}$ exists. The scalar potential $\psi$ is

$$
\psi=\frac{1}{2 \pi} \int B_{n}\left(x^{\prime}, y^{\prime}\right) G\left(x, y, z, x^{\prime}, y^{\prime}\right) d x^{\prime} d y^{\prime},
$$

where $G=1 / \sqrt{\left|\boldsymbol{r}^{\prime}-\boldsymbol{r}\right|}$. The scalar function is determined automatically by the normal component of the observed magnetic field, whereas $\boldsymbol{B}=0$ is assumed as $\boldsymbol{r}$ approaches $\infty$. This method can be applied to an isolated active region that is not influenced by the magnetic fields of other regions. On the other hand, if the magnetic field lines in the active region extend into another active region, the boundary conditions at the sides and top are no longer appropriate.

The Fourier expansion can be used for deriving the solution of Eqs. (5) and (6). The solution was presented by Priest (2014), as follows:

$$
\begin{aligned}
& B_{x}=-\sum_{\boldsymbol{k}} \frac{k_{x}}{|k|} B_{\boldsymbol{k}} e^{i k_{x}+i k_{y}-k z,} \\
& B_{y}=-\sum_{\boldsymbol{k}} \frac{k_{y}}{|k|} B_{\boldsymbol{k}} e^{i k_{x}+i k_{y}-k z,} \\
& B_{z}=B_{0}+\sum_{\boldsymbol{k}} B_{\boldsymbol{k}} e^{i k_{x}+i k_{y}-k z,}
\end{aligned}
$$

where the bottom boundary values are expanded into Fourier components $k_{x}$ and $k_{y}$. This formulation implies that all of the components decay exponentially, implying $\boldsymbol{B}=0$ at $z=\infty$. However, the side boundaries automatically obey periodic boundary conditions, so this method is useful only for describing areas far from the side boundaries.

We can easily extend Eq. (6) in spherical coordinates $(r, \theta, \phi)$ and thus obtain a solution for the whole sun, as shown in Fig. 2b. This overcomes the problem mentioned above regarding the connectivity of the field lines. In spherical coordinates, the solution to Eq. (6) can be written using Legendre polynomials (Altschuler and Newkirk 1969), as follows:

$$
\psi=\sum_{n=1} \sum_{m=0}\left[\left(\frac{1}{r}\right)^{n+1}\left(g_{n}^{m} \cos \phi+h_{n}^{m} \sin \phi\right) P_{n}^{m}(\cos \theta)\right],
$$

where $P_{n}^{m}(\cos \theta)$ are Legendre polynomials, and $g_{n}^{m}$ and $h_{n}^{m}$ are coefficients obtained from spherical harmonics analysis. The boundary condition is based on the normal component of the photospheric magnetic field, and the Neumann condition of $\psi$ is the same as that in Eq. (7). Using the above calculations, the potential fields can be expressed as follows:

$$
\begin{aligned}
& B_{r}=-\sum_{n=1}^{N} \sum_{m=0}^{n}\left\{(n+1)\left(\frac{1}{r}\right)^{n+2}\left(g_{n}^{m} \cos \phi+h_{n}^{m} \sin \phi\right) P_{n}^{m}(\cos \theta)\right\}, \\
& B_{\theta}=\sum_{n=1}^{N} \sum_{m=0}^{n}\left\{\left(\frac{1}{r}\right)^{n+2}\left(g_{n}^{m} \cos \phi+h_{n}^{m} \sin \phi\right) \frac{d P_{n}^{m}(\cos \theta)}{d \theta}\right\}, \\
& B_{\phi}=-\sum_{n=1}^{N} \sum_{m=0}^{n}\left\{m\left(\frac{1}{r}\right)^{n+2}\left(g_{n}^{m} \sin \phi-h_{n}^{m} \cos \phi\right) \frac{P_{n}^{m}(\cos \theta)}{\sin \theta}\right\} .
\end{aligned}
$$

As an example, one result is shown in Fig. 2b, which can be used to depict the field lines covering the sun. 
One advantage of the potential field extrapolation method is that the solution is relatively easily obtained; there are several techniques for doing this. On the other hand, the potential field is a minimum energy state that does not store the free magnetic energy released in the solar flares. This implies that the observed field lines in the area close to the PIL cannot be captured by the potential field. To convert the potential field into the dynamic phase of the solar flares, it is necessary to obtain the Poynting flux through the photosphere in order to obtain the free energy (Feynman and Martin 1995; van Ballegooijen and PMartens 1989).

\section{Linear force-free field}

The force-free Eq. (1) can be rewritten as

$$
\nabla \times \boldsymbol{B}=\alpha \boldsymbol{B},
$$

where $\alpha$ is a coefficient. After taking the divergence of this equation, the left-hand side vanishes, and thus, we have

$$
(\boldsymbol{B} \cdot \nabla) \alpha=0,
$$

which implies that the coefficient $\alpha$ is constant along all field lines. If the coefficient $\alpha$ is constant everywhere (not only along the field lines), Eq. (13) becomes a linear Equation that can be reduced to the Helmholtz equation,

$$
\nabla^{2} \boldsymbol{B}+\alpha \boldsymbol{B}=0
$$

by taking the curl of Eq. (13). We call this solution the linear force-free field (LFFF), and it is also specified with an appropriate boundary condition.

For example, (Chiu and Hilton 1977) found the analytical general solution by using Green's functions:

$$
B_{i}=\frac{1}{2 \pi} \int_{-\infty}^{\infty} d x^{\prime} d y^{\prime}\left[\overline{G_{i}}\left(\boldsymbol{x}, \boldsymbol{x}^{\prime}\right) B_{z}\left(x^{\prime}, y^{\prime}, 0\right)+\tilde{G}_{i}\left(\boldsymbol{x}, \boldsymbol{x}^{\prime}\right) C\left(x^{\prime}, y^{\prime}\right)\right],
$$

where $C\left(x^{\prime}, y^{\prime}\right)$ is any finite integrable function (see Chiu and Hilton (1977)). $\tilde{G}_{i}\left(\boldsymbol{x}, \boldsymbol{x}^{\prime}\right)$ is defined as

$$
\begin{aligned}
& \overline{G_{x}}=\frac{x-x^{\prime}}{R} \frac{\partial \bar{\Gamma}}{\partial z}+\alpha \bar{\Gamma} \frac{y-y^{\prime}}{R}, \\
& \overline{G_{y}}=\frac{y-y^{\prime}}{R} \frac{\partial \bar{\Gamma}}{\partial z}-\alpha \bar{\Gamma} \frac{x-x^{\prime}}{R}, \\
& \overline{G_{z}}=-\frac{\bar{\Gamma}}{\partial R}-\frac{\bar{\Gamma}}{R},
\end{aligned}
$$

where $R=\left(x-x^{\prime}\right)^{2}+\left(y-y^{\prime}\right)^{2}, \bar{\Gamma}$ is

$$
\bar{\Gamma}=\frac{z}{R r} \sin (\alpha r)-\frac{1}{R} \sin (\alpha z),
$$

and $r=\left(x-x^{\prime}\right)^{2}+\left(y-y^{\prime}\right)^{2}+z^{2}$. Using these equations, if we are given $B_{z}$ and the force-free $\alpha$ at the photosphere, then the LFFF is automatically determined.
Unlike the potential field, the LFFF can yield the free magnetic energy. In general, however, the observed forcefree $\alpha$ measured in the photosphere varies in space. In particular, in solar active regions, the coefficient $\alpha$ attains high values close to the PIL and small values far from the PIL. This implies that the LFFF is inappropriate for modeling solar active regions. Therefore, we need to obtain the NLFFF extrapolation by using the observed force-free $\alpha$, i.e., we need to obtain not only the normal component of the magnetic field but also the horizontal components at the photosphere in order to reproduce the magnetic field of a solar active region.

\section{Nonlinear force-free field}

To demonstrate suitable magnetic fields in the solar active region, we consider solving the force-free Eq. (1) directly. However, because this equation contains nonlinearities that cannot be solved analytically, numerical techniques are necessary (i.e., Schrijver et al. (2006) or Metcalf et al. (2008)). Since important information can be obtained from observed photospheric magnetic fields, this becomes a boundary value problem. Below, we briefly describe several numerical methods that have been developed.

Vertical integration method. The algorithm of the vertical integration method is quite simple. The magnetic fields are integrated upward in the $z$ direction, as originally proposed by Nakagawa (1974) and further extended by Wu et al. (1990). Under the force-free assumption, the current densities of the horizontal components along the solar surface can be calculated as follows:

$$
\begin{gathered}
J_{x 0}=\alpha_{0} B_{x 0}, \\
J_{y 0}=\alpha_{0} B_{y 0},
\end{gathered}
$$

where $B_{x 0}$ and $B_{y 0}$ are the horizontal components of the photospheric magnetic field, $J_{x 0}$ and $J_{y 0}$ are the horizontal components of the current density, and $\alpha_{0}$ is the forcefree alpha obtained from $J_{z 0} / B_{z 0}$. Using Ampere's law, Eq. (3), and the solenoidal condition, Eq. (2), the following equations are obtained for the $\mathrm{z}$-derivatives of the magnetic field:

$$
\begin{aligned}
& \frac{\partial B_{x}}{\partial z}=J_{y 0}+\frac{\partial B_{z 0}}{\partial x}, \\
& \frac{\partial B_{y}}{\partial z}=\frac{\partial B_{z 0}}{\partial y}-J_{x 0}, \\
& \frac{\partial B_{z}}{\partial z}=-\frac{\partial B_{x 0}}{\partial x}-\frac{\partial B_{y 0}}{\partial y} .
\end{aligned}
$$

The integration, in which the information about the photospheric magnetic field is extended upward, is repeated, and the coronal magnetic field can be calculated in 3D. However the above algorithm is mathematically ill-posed, i.e., the calculation is not robust, as has been reported in several papers (e.g., Wiegelmann and Sakurai (2012)). For 
instance, once the nonphysical phenomena due to numerical errors appear during the integration, the magnetic field increases exponentially. One reason for this is that no restrictions are imposed on the top and side boundaries.

The Green's function method. A similar mathematical approach that uses the Green's function was developed by Yan (1995) and Yan and Sakurai (2000) but the magnetic field is assumed as follows: $\boldsymbol{B}=O\left(\frac{1}{r^{2}}\right)$, i.e., $\boldsymbol{B}=0$ as $r=>\infty$. They found the NLFFF solution based on Green's second identity, as follows:

$$
c_{i} \boldsymbol{B}_{i}=\int_{S}\left(\boldsymbol{Y} \frac{d \boldsymbol{B}}{d n}-\frac{d \boldsymbol{Y}}{d n} \boldsymbol{B}_{0}\right) d S,
$$

where $c_{i}=1$ and $c_{i}=1 / 2$ correspond to points in the volume and at the boundary, respectively, $\boldsymbol{B}_{0}$ is the measured photospheric magnetic field, and $\boldsymbol{Y}$ is a reference function,

$$
\boldsymbol{Y}(r)=\frac{\cos \left(\lambda_{i} \boldsymbol{r}\right)}{4 \pi\left|\boldsymbol{r}-\boldsymbol{r}^{\prime}\right|},
$$

where $r^{\prime}$ is a fixed point and $\lambda\left(r^{\prime}\right)$ is a parameter that depends on $r^{\prime}$. The reference function satisfies the Helmholtz equation,

$$
\nabla^{2} \boldsymbol{Y}+\lambda^{2} \boldsymbol{Y}=\delta_{i},
$$

where $\delta_{i}$ is the Dirac delta function. The parameter $\lambda_{i}$ can be obtained by solving

$$
\int_{V} Y_{i}\left(\lambda_{i}^{2} B_{i}-\alpha^{2} B_{i}-\left(\nabla \alpha \times \boldsymbol{B}_{i}\right)\right) d V=0 .
$$

Although it has been pointed out that this technique is slow (Wiegelmann and Sakurai 2012), recently, the calculation speed has been dramatically accelerated by using a GPU (Wang et al. 2013).

Grad-Rubin method. Sakurai (1981) was the first to use the Grad-Rubin method for calculating the magnetic field in solar active regions, and this method was later extended, e.g., Amari et al. (2006). This technique follows directly from the force-free field property. First, the potential field is calculated based only on the normal components of the magnetic field. The force-free $\alpha$ can be measured at the bottom surface as $\alpha=J_{z} / B_{z}$, and it can be distributed in $3 \mathrm{D}$ according to the following equation:

$$
\boldsymbol{B}^{k} \cdot \nabla \alpha^{k}=0,
$$

where $k$ is the iteration number and $B^{0}$ corresponds to the potential field. The magnetic field is updated according to

$$
\nabla \times \boldsymbol{B}^{k+1}=\alpha^{k} \boldsymbol{B}^{k}
$$

and

$$
\nabla \cdot B^{k+1}=0 .
$$

Since the vector potential $\boldsymbol{A}^{k}$ satisfying $\nabla \times \boldsymbol{A}^{k}=\boldsymbol{B}^{k}$ can be written as

$$
A^{k+1}=\int \frac{J^{k}}{\sqrt{\left(x-x^{\prime}\right)^{2}+\left(y-y^{\prime}\right)^{2}+\left(z-z^{\prime}\right)^{2}}} d x^{\prime} d y^{\prime} d z^{\prime}
$$

the updated $\boldsymbol{B}$ automatically satisfies the solenoidal condition, and it is then substituted back into Eq. (23). This process is repeated until the magnetic field reaches a steady state. Although the force-free $\alpha$ can be determined at positive or negative polarity and will satisfy Eq. (23), the single-polarity information is neglected. Nevertheless, Régnier et al. (2002) and Canou and Amari (2010) were able to reconstruct magnetic fields that agree with the observations.

Recently, the Grad-Rubin method has been improved by Amari et al. (2010); Wheatland and Régnier (2009), and (Wheatland and Leka 2011), who have obtained the unique solution by using two different solutions derived from different polarities, i.e., by changing the distribution of the force-free $\alpha$ at the bottom surface.

$M H D$ relaxation method. In the MHD relaxation methods, the MHD equations are solved directly (in particular, this is the zero-beta MHD approximation (Mikić et al. 1988)); they solved

$$
\begin{aligned}
& \frac{\partial \boldsymbol{v}}{\partial t}=-(\boldsymbol{v} \cdot \nabla) \boldsymbol{v}+\boldsymbol{J} \times \boldsymbol{B}+v \nabla^{2} \boldsymbol{v}, \\
& \frac{\partial \boldsymbol{B}}{\partial t}=\nabla \times(\boldsymbol{v} \times \boldsymbol{B}-\eta \boldsymbol{J}),
\end{aligned}
$$

and

$$
\boldsymbol{J}=\nabla \times \boldsymbol{B}
$$

to find the force-free solution while keeping the photospheric magnetic field as the boundary condition. Here, $v$ is the plasma velocity, and $v$ and $\eta$ are the viscosity and resistivity, respectively. The zero-beta MHD is an extreme approximation of the low-beta solution. However, since a force-free state can be assumed in the zero-beta approximation, this method is valid. Several studies (Mikić and McClymont 1994; McClymont and Mikic 1994; Jiang and Feng 2012; Inoue et al. 2014b) have employed the potential field as the initial condition; consequently, the magnetic twist on the bottom surface is obtained by replacing the tangential components of the photospheric magnetic field above which the magnetic fields relaxes toward the force-free state through the MHD relaxation process. This process is called the stress-and-relaxation method (Roumeliotis 1996). In a simpler treatment, known as the magnetofrictional method, the equation of motion (27) is replaced with

$$
\boldsymbol{v}=\mu \boldsymbol{J} \times \boldsymbol{B},
$$


where $\mu$ is a coefficient. This technique can also be used to find the force-free solution (Valori et al. 2005), and it has been applied to the photospheric magnetic field.

Note that if the three components of the photospheric magnetic field are fully satisfied at the solar surface and if the plasma velocity is zero there, these conditions are not consistent with the induction equation, which requires information about the differential value in the normal direction. Consequently, an error appears in $\nabla \cdot \boldsymbol{B}$. Therefore, the errors arising during the relaxation process should be eliminated, and several methods have been developed for eliminating them (Tóth 2000; Miyoshi and Kusano 2011). Often, the projection method is used, and this removes the errors derived from the potential component. We decompose the numerically obtained magnetic field $\boldsymbol{B}_{N}$ into $\boldsymbol{B}_{p}$ (the potential component) and $\boldsymbol{B}_{n p}$ (the non-potential component), as follows:

$$
\boldsymbol{B}_{N}=\boldsymbol{B}_{p}+\boldsymbol{B}_{n p}
$$

In general, a vector field $\boldsymbol{B}$ can be described as

$$
\boldsymbol{B}=\nabla \psi_{p}+\nabla \times \boldsymbol{A}_{n p},
$$

where $\psi_{p}$ and $\boldsymbol{A}_{n p}$ are the scalar and vector potentials, respectively. Taking into account Eq. (5), $\nabla \psi_{p}$ and $\nabla \times$ $\boldsymbol{A}_{n p}$ correspond, respectively, to the potential and nonpotential components of the magnetic field. Taking the divergence of Eq. (32), the equality $\nabla \cdot \nabla \times \boldsymbol{A}_{n p}=\nabla \cdot \boldsymbol{B}_{n p}=0$ is automatically satisfied. However, it is not guaranteed that $\nabla \cdot \nabla \psi_{p}=\nabla \cdot \boldsymbol{B}_{p}=0$. If $\boldsymbol{B}_{p}$ contains a numerical error, we further decompose it into $\boldsymbol{B}_{p^{\prime}}$, which satisfies the solenoidal condition, and $\boldsymbol{B}_{\text {error }}$, the error, as follows:

$$
\boldsymbol{B}_{p}=\boldsymbol{B}_{p^{\prime}}+\boldsymbol{B}_{\text {error }}
$$

where, in general, $\boldsymbol{B}_{\text {error }}$ does not meet the solenoidal condition. However, taking the divergence of Eq. (33), the equation can be reduced to the Poisson equation,

$$
\nabla \cdot \boldsymbol{B}_{\text {error }}=\nabla \cdot \boldsymbol{B}_{N}=\nabla^{2} \psi_{p} .
$$

Consequently, this equation can be solved, and the magnetic field satisfying the solenoidal condition $\boldsymbol{B}^{\prime}$ can be updated as follows:

$$
\boldsymbol{B}^{\prime}=\boldsymbol{B}_{N}-\nabla \psi_{p}
$$

This technique has been widely used for eliminating errors (Tanaka 1995; Tóth 2000); however, solving the Poisson equation is computationally demanding. Therefore, numerical techniques for improving the calculation speed, e.g., a multigrid technique, are required (Inoue et al. 2014b).

Another technique was proposed by Dedner et al. (2002), who introduced a modified induction equation,

$$
\frac{\partial \boldsymbol{B}}{\partial t}=\nabla \times(\boldsymbol{v} \times \boldsymbol{B}-\eta \boldsymbol{J})-\nabla \phi,
$$

and a convenient equation for eliminating the errors derived from $\nabla \cdot \boldsymbol{B}$,

$$
\frac{\partial \phi}{\partial t}+c_{h}^{2} \nabla \cdot \boldsymbol{B}=-\frac{c_{h}^{2}}{c_{p}^{2}} \phi,
$$

together with the equation of motion (27) and Ampere's law (29). Using Eq. (36), Eq. (37) can be changed to

$$
\frac{\partial^{2}(\nabla \cdot \boldsymbol{B})}{\partial t^{2}}+\frac{c_{h}^{2}}{c_{p}^{2}} \frac{\partial(\nabla \cdot \boldsymbol{B})}{\partial t}=c_{h}^{2} \nabla^{2}(\nabla \cdot \boldsymbol{B}),
$$

where $c_{h}$ and $c_{p}$ correspond to the advection and diffusion coefficients; this plays a role in propagating and diffusing the numerical errors of $\nabla \cdot \boldsymbol{B}$. The main advantage of this method is that it can be implemented very easily without significantly changing the numerical code. Another advantage is that this method is less computationally demanding than the projection method. These advantages were demonstrated by Inoue et al. (2014b).

The vector potential is specified to maintain the solenoidal condition. Using the vector potential, the induction equation can be written as

$$
\frac{\partial \boldsymbol{A}}{\partial t}=-\boldsymbol{E}-\nabla \Phi,
$$

where $\boldsymbol{E}=\eta \boldsymbol{J}-\boldsymbol{v} \times \boldsymbol{B}$ and $\Phi$ is the gage. Several papers have used the NLFFF extrapolation (e.g., van Ballegooijen et al. (2000) and Cheung and DeRosa (2012)). In this case, the solution is sought under the proper boundary conditions and gage. Simply, $B_{z}$ and $J_{z}$ are fixed at the boundary (i.e., $A_{x}$ and $A_{y}$ are fixed), then $A_{z}$ is obtained from $\nabla^{2} \boldsymbol{A}=\boldsymbol{J}$ under the Coulomb gage $\nabla \cdot \boldsymbol{A}=0$. A solution obtained by this method will completely satisfy the solenoidal condition. On the other hand, there is no guarantee that the horizontal components at the bottom surface, which are obtained by iteration, will match observed values.

The constrained transport (CT) method (Brackbill and Barnes 1980; Evans and Hawley 1988) uses a numerical differential approach to maintaining the solenoidal condition. When the magnetic field $\boldsymbol{B}$ and electric field $\boldsymbol{E}$ are defined at the face center and edge centers of each numerical cell, i.e.,

$$
\begin{aligned}
\frac{d}{d t} B_{x i+1 / 2, j} & =-\frac{E_{z i+1 / 2, j+1 / 2}-E_{z i+1 / 2, j-1 / 2}}{\Delta y}, \\
\frac{d}{d t} B_{y i, j+1 / 2} & =\frac{E_{z i+1 / 2, j+1 / 2}-E_{z i-1 / 2, j+1 / 2}}{\Delta x},
\end{aligned}
$$

where symmetry is assumed in the $\mathrm{z}$ direction, then the solenoidal condition is automatically satisfied:

$$
\frac{d}{d t}\left(\frac{B_{x i+1 / 2, j}-B_{x i-1 / 2, j}}{\Delta x}+\frac{B_{y i, j+1 / 2}-B_{y i j-1 / 2}}{\Delta y}\right)=0 .
$$

However, the solenoidal condition requires consistent interaction with the boundary condition, and thus, it 
might be difficult to use it with the NLFFF calculations, which require the three components of the photospheric magnetic field.

Optimization method. Wheatland et al. (2000) proposed an optimization method that was later improved by Wiegelmann (2004). This method iteratively minimizes a function $L$ related to $\boldsymbol{J} \times \boldsymbol{B}$ and $\nabla \cdot \boldsymbol{B}$. First, we define a function $L$ as

$$
L=\int\left(\frac{1}{B^{2}}|(\nabla \times \boldsymbol{B}) \times \boldsymbol{B}|^{2}+|\nabla \cdot \boldsymbol{B}|^{2}\right) d V
$$

it is the sum of the Lorentz force and the solenoidal condition, and its value is prescribed to be zero in order to satisfy the force-free condition. The time derivative is expressed as

$$
\frac{1}{2} \frac{d L}{d t}=-\int \frac{\partial \boldsymbol{B}}{\partial t} \cdot \boldsymbol{F} d V-\int \frac{\partial \boldsymbol{B}}{\partial t} \cdot \boldsymbol{G} d S
$$

where $\boldsymbol{F}$ and $\boldsymbol{G}$ are high-order differential equations in terms of $\boldsymbol{B}$. If the function $\boldsymbol{F}$ satisfies

$$
\frac{\partial \boldsymbol{B}}{\partial t}=\mu \boldsymbol{F}
$$

and if the magnetic fields on the surface vanish at infinity, then the $L$ monotonically decreases. The problem is then reduced to iteratively finding the steady state the time-dependent magnetic field $\boldsymbol{B}$ that satisfies Eq. (44).

NLFFF extrapolation using the observed images. van Ballegooijen (2004) modeled a filament by inserting a twisted magnetic flux tube, whose axis was along the observed filament, into a potential field, with the magnetofriction (van Ballegooijen et al. 2000) driving the system toward the force-free state. In this case, although the horizontal fields were not used, the filament and the sigmoid structure were satisfactorily reproduced (Bobra et al. 2008; Su et al. 2009; Savcheva et al. 2012). Rather than using the methods accounting for the photospheric horizontal fields, modeling the filaments in the quiet region would be very useful because the values are very weak and the directions are random, so this might depend on the observations. In an attempt to obtain consistent magnetic fields, several studies have considered the topology of the coronal loops obtained from images, in addition to accounting for the photospheric magnetic field (Aschwanden et al. 2014; Malanushenko et al. 2014).

Unfortunately, the NLFFF does not allow the full calculation of the coronal magnetic fields. First of all, because, in general, the photospheric magnetic field cannot satisfy the force-free state, there is a contradiction between the bottom and inner regions; consequently, the 3Dreconstructed field also deviates from the force-free state. Furthermore, although several methods have been developed for exploring the NLFFF, there are no guarantees that there is a unique solution that fits the photospheric magnetic field applied to a given boundary condition. In the
NLFFF approach, there are several open problems related to the free magnetic energy or the topologies of the magnetic fields (Schrijver et al. 2008; De Rosa et al. 2009). Thus, there is a need for confirmation of the reliability of this approach.

\section{NLFFF extrapolation applied to a reference field (Low and Lou 1990)}

The above methods for the NLFFF reconstruction have been applied to the photospheric magnetic field observed in the solar active region. Most of these methods required knowledge of the reference magnetic field in order to determine to what extent the reconstructed field approaches the force-free state. One of the widely known solutions is a semi-analytical force-free field that was presented by (Low and Lou 1990). These authors found a force-free solution in spherical coordinates, where symmetry was assumed in the $\phi$ direction:

$$
\boldsymbol{B}=\frac{1}{r \sin \theta}\left(\frac{1}{r} \frac{\partial A}{\partial \theta} \boldsymbol{r},-\frac{\partial A}{\partial r} \theta, Q \phi\right),
$$

where $\mathrm{A}$ and $\mathrm{Q}$ are functions of $r$ and $\theta$. The force-free Eq. (1) can be rewritten as

$$
\frac{\partial^{2} A}{\partial r^{2}}+\frac{1-\mu^{2}}{r^{2}} \frac{\partial^{2} A}{\partial \mu^{2}}+Q \frac{d Q}{d A}=0
$$

where $\mu=\cos (\theta)$ and $\alpha=d Q / d A$. It can be further rewritten as

$$
\left(1-\mu^{2}\right) \frac{\partial^{2} P}{\partial \mu^{2}}+n(n+1) P+a^{2} \frac{1+n}{n} P^{1+2 / n}=0,
$$

using a separable solution

$$
A(r, \theta)=\frac{P(\mu)}{r^{n}},
$$

and as

$$
Q(A)=a A^{1+2 / n} .
$$

These formulas are obtained under the assumption of a vanishing magnetic field as $r \rightarrow 0$, i.e., for positive $n$. Although we can write down the 1D differential equation with respect to $P(\mu)$, shown as Eq. (47), it cannot be solved analytically due to its nonlinearity. The solution of this equation, therefore, is obtained numerically. The boundary condition is that $P=0$ at $\mu=-1$ and 1 , which was originally set by Low and Lou (1990), and the solution is called the Low and Lou solution. The boundary conditions are that $B_{\theta}$ and $B_{\phi}$ vanish along the axis, and the differential equation can be solved as a boundary value problem. One of the solutions is shown in Fig. 5a; here, the solution was transformed to Cartesian coordinates, and $n=1$ and $a^{2}=0.425$ are assumed (see Low and Lou (1990) for details). The accuracy of the NLFFF was checked using this solution as the reference magnetic field. 

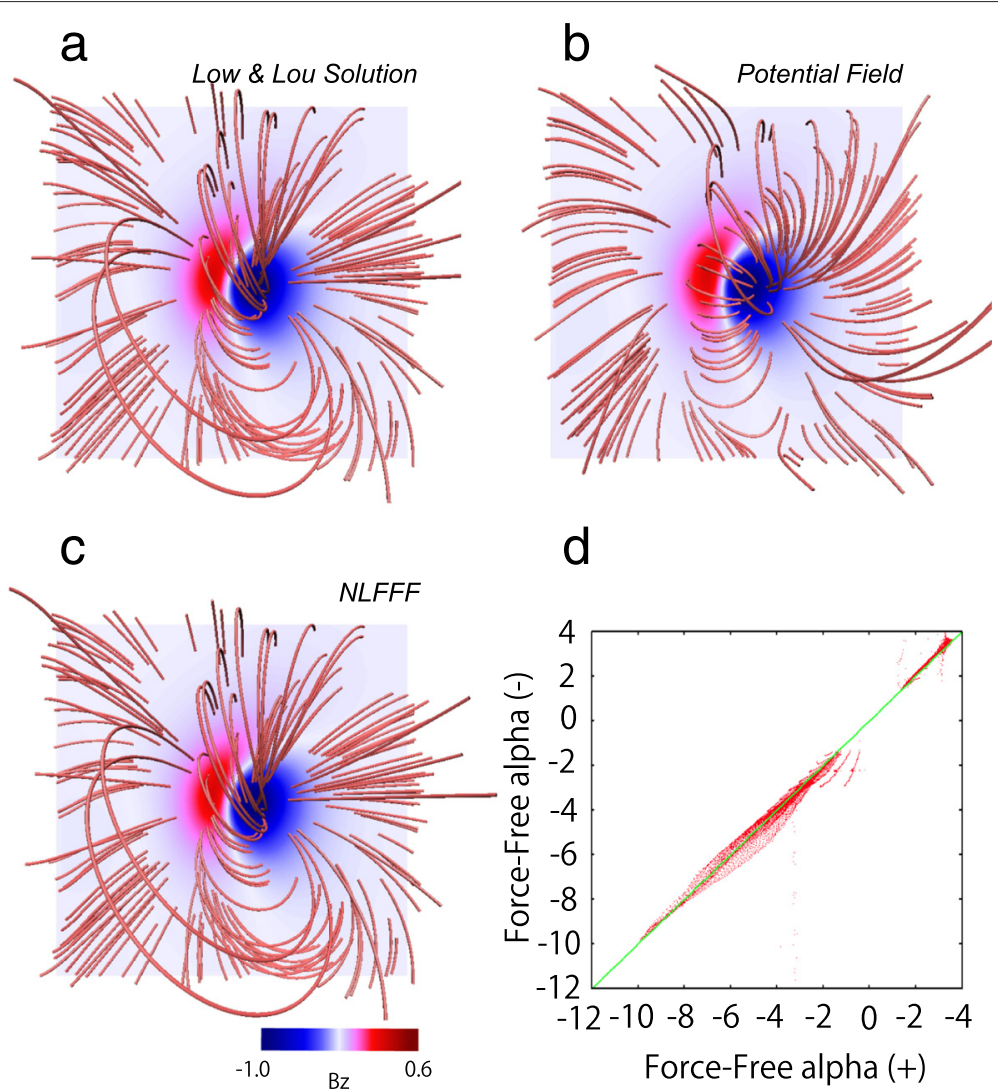

Fig. 5 Semi-analytical Low and Lou solution and the NLFFF solution. a The magnetic field lines of the Low and Lou solution with the $B_{z}$ distribution are shown in blue and red. $\mathbf{b}$ The potential field extrapolated from only the normal component of the magnetic field, using the Low and Lou solution on all boundaries. c The NLFFF solution based on the MHD relaxation method (Inoue et al. 2014b), extrapolated from all three components of the magnetic field of the Low and Lou solution on all boundaries. $\mathbf{d}$ Distribution of the force-free $\alpha$ from Inoue et al. 2014b, where the horizontal and vertical axes correspond to the force-free $\alpha$ measured at the field lines footpoints. The green line has a slope of unity (i.e., $y=x$ ). The image in (d) is copyright AAS and is reproduced by permission

Schrijver et al. (2006) estimated the accuracy of the NLFFF as reconstructed by various different methods; this included a semi-analytical force-free solution introduced by Low and Lou (1990). Their results suggest that the reconstruction accuracy is strongly method dependent, i.e., several methods satisfactorily captured the Low and Lou solution, although other methods failed. On the other hand, during the past decade, many efforts have been made to improve the numerical code for the NLFFF reconstruction (Amari et al. 2006; Valori et al. 2007; He and Wang 2008; Wheatland and Leka 2011; Jiang and Feng 2012; Inoue et al. 2014b).

Below, we review the results based on a recent extrapolation method that was proposed by Inoue et al. (2014b) and is based on the MHD relaxation method. The potential field was reconstructed, based only on the normal component of the boundary magnetic field. This result is shown in Fig. 5b and differs significantly from the Low and Lou solution. Next, the reconstructed horizontal fields at the bottom surface were replaced by those of the Low and Lou solution, following which the magnetic fields in the domain were iteratively relaxed according to the equation of motion (27), the induction Eq. (36), Amperes law (29), and Eq. (37), which was used to correct the errors in $\nabla \cdot \boldsymbol{B}$.

During the iterations, at all boundaries, the vector $\boldsymbol{B}$ was fixed to be equal that in the Low and Lou solution, the velocity was set to zero, and the Neumann condition was imposed on $\phi$, i.e., $\partial \phi / \partial n=0$, where $n$ is the direction perpendicular to the boundaries. In order to avoid a large discontinuity between the bottom and the inner domain, the velocity field was adjusted as follows. We defined $v^{*}=$ $|\boldsymbol{v}| /\left|\boldsymbol{v}_{A}\right|$, and if $v^{*}$ became larger than $v_{\max }$, the velocity was modified as follows:

$$
\boldsymbol{v} \Rightarrow \frac{v_{\max }}{v^{*}} \boldsymbol{v}
$$

where, $v_{\max }=1.0$. The resistivity was given as follows: 


$$
\eta=\eta_{0}+\eta_{1} \frac{|\boldsymbol{J} \times \boldsymbol{B}||\boldsymbol{v}|}{|\mathbf{B}|}
$$

where $\eta_{0}=3.75 \times 10^{-5}$ and $\eta_{1}=1.0 \times 10^{-3}$ (both are non-dimensional). The second term was introduced to accelerate the relaxation to the force free field, particularly in a weak-field region. In this study, $c_{h}^{2}$ and $c_{p}^{2}$ were set to 5.0 and 0.1 , respectively; these values were selected by trial and error and depend on the boundary conditions, but it is best if the value of $c_{h}$ is first set to account for the CFL condition. The viscosity was assumed as $v=1.0 \times 10^{3}$; the viscosity also plays an important role in smoothly connecting the boundaries and nearby inner region, which indirectly helps our MHD calculation. A more detailed explanation of this was presented by Inoue et al. (2014b).

Eventually, the final state obtained by using this method almost completely reproduced the Low and Lou solution, as shown in Fig. 5c. Quantitative results were also presented. The force-free $\alpha$ was measured at both footpoints of all field lines, and this is shown in Fig. 5d. The forcefree $\alpha$ must be constant along the field lines, following Eq. (14), and from Fig. 5d, it can be concluded that this relation is satisfied. In addition, the authors quantitatively evaluated the accuracy by following Schrijver et al. (2006), evaluating

$$
\begin{array}{r}
C_{\mathrm{vec}}=\frac{\sum_{i} \boldsymbol{B}_{i} \cdot \boldsymbol{b}_{i}}{\sum_{i}\left|\boldsymbol{B}_{i}\right|^{2} \sum_{i}\left|\boldsymbol{b}_{i}\right|^{2}}, \\
C_{\mathrm{Cs}}=\frac{1}{N} \sum_{i} \frac{\boldsymbol{B}_{i} \cdot \boldsymbol{b}_{i}}{\left|\boldsymbol{B}_{i}\right|\left|\boldsymbol{b}_{i}\right|}, \\
1-E_{\mathrm{M}}=\frac{1}{N} \sum_{i} \frac{\left|\boldsymbol{b}_{i}-\boldsymbol{B}_{i}\right|}{\left|\boldsymbol{B}_{i}\right|}, \\
1-E_{\mathrm{N}}=\frac{\sum_{i}\left|\boldsymbol{b}_{i}-\boldsymbol{B}_{i}\right|}{\sum_{i}\left|\boldsymbol{B}_{i}\right|}, \\
\epsilon=\frac{\sum_{i}\left|\boldsymbol{b}_{i}\right|^{2}}{\sum_{i}\left|\boldsymbol{B}_{i}\right|^{2}},
\end{array}
$$

where $\boldsymbol{B}$ and $\boldsymbol{b}$ are Low and Lou solution (reference solution) and the extrapolated solution, respectively, $C_{\text {vec }}$ is the vector correlation, $C_{c s}$ is the Cauchy-Schwarz inequality, $E_{M}$ is the mean vector error, $E_{N}$ is the normalized vector error, $\epsilon$ is the energy ratio, and $N$ is the number of vectors in the field. Inoue et al. (2014b) obtained $C_{\mathrm{vec}}=1.0, C_{\mathrm{cs}}=1.0,1-E_{\mathrm{N}}=0.97,1-E_{\mathrm{M}}=0.95$, $\epsilon=1.02$, and these values were estimated over the entire region, which was divided into $64 \times 64 \times 64$ grids (see Inoue et al. 2014b for details). They confirmed that the NLFFF can be reconstructed with high accuracy. Most of the recently developed methods allow for the recording of these values. Thus, it is possible to achieve force-free field extrapolation if the boundary condition completely satisfies the force-free condition.

\section{NLFFF extrapolation applied to the solar active region 3D magnetic fields in the solar active region}

In contrast to the NLFFF extrapolation using the Low and Lou solution, some problems arise when the bottom boundary is applied to the photospheric magnetic field. Schrijver et al. (2008) performed the NLFFF extrapolations by using the photospheric magnetic field observed by the Hinode satellite, corresponding to the period of $6 \mathrm{~h}$ before the X3.4-class flare that occurred in the solar active region 10930 on 13 December 2006. Different methods were applied for the NLFFF extrapolation. The authors pointed out a method-dependent accumulation of the free magnetic energy in the NLFFF. According to their calculations, a single NLFFF could yield sufficient free magnetic energy to produce an X-class flare. De Rosa et al. (2009) also performed the NLFFF extrapolation using different methods and for a different another active region (AR10953). They reported method-dependent configurations of the magnetic fields. From these results, it appeared that the NLFFF required further development.

Although the NLFFF remains problematic and does not enable the complete reproduction of the coronal magnetic field on the basis of photospheric data, several recent studies had roughly captured the field lines observed in EUV images, as well as processes involving stored-andreleased magnetic energy, helicity, and flares (e.g., Canou and Amari (2010); Inoue et al. (2013); Vemareddy et al. (2013); Jiang and Feng (2013); Malanushenko et al. (2014); Aschwanden et al. (2014); Amari et al. (2014).

In what follows, we describe NLFFF results based on the MHD relaxation method developed by Inoue et al. (2014b); note that the above equations are identical to those used by Low and Lou. The potential field is first reconstructed as the initial condition, and the boundary conditions are almost identical to those in the previous calculation, except that the potential fields are now fixed at the side and top boundaries. The following procedure is used to determine the bottom boundary. During the iterative process, the transverse components $\left(\boldsymbol{B}_{\mathrm{BC}}\right)$ at the bottom boundary are evaluated according to

$$
\boldsymbol{B}_{\mathrm{BC}}=\zeta \boldsymbol{B}_{\mathrm{obs}}+(1-\zeta) \boldsymbol{B}_{\text {pot }},
$$

where $\boldsymbol{B}_{\mathrm{obs}}$ and $\boldsymbol{B}_{\text {pot }}$ are the transverse components of the observational and the potential field, respectively, and $\zeta$ is a coefficient ranging from 0 to $1 . R$ is introduced as an indication parameter for the force-free state, defined as $R=\int|\boldsymbol{J} \times \boldsymbol{B}|^{2} d V$; when it drops below a critical value, denoted by $R_{\min }$, then $\zeta$ increases as $\zeta=\zeta+d \zeta$, where $\mathrm{d} \zeta$ is given as a parameter. As $\zeta$ approaches unity, $\boldsymbol{B}_{\mathrm{BC}}$ becomes consistent with the observational data. The vector fields include spurious forces that produce a sharp jump from the photosphere to the interior domain, and the above process can help to reduce their effects. In this study, $R_{\min }=5.0 \times 10^{-3}, d \zeta=0.02$, and $v_{\max }=0.01$. In 
the MHD equations, $c_{h}^{2}$ and $c_{p}^{2}$ are given as constant values, 0.04 and 0.1 , respectively, and $v=1.0 \times 10^{-3}$. The resistivity is included in Eq. (51), with $\eta_{0}=5.0 \times 10^{-5}$ and $\eta_{1}=1.0 \times 10^{-3}$. For further details, see Inoue et al. (2014b).

Figure 6a shows the photospheric magnetic field $90 \mathrm{~min}$ before the M6.6-class flare that occurred on 13 February 2011. These data were obtained by a helioseismic and magnetic imager (HMI; Scherrer et al. (2012)) onboard the solar dynamics observatory (SDO) satellite (Pesnell et al. 2012). The upper and lower panels in Fig. $6 \mathrm{~b}$ show enlarged views of the central area in Fig. 6a; the arrows derived from the horizontal magnetic fields in the potential field are shown in the upper panel, and those derived from the observed one are shown in the lower panel. Figure $6 c$, d shows the magnetic field lines in the potential field and in the NLFFF approximation, respectively, superimposed on Fig. 6a. In particular, the central part of the NLFFF, in which strong sheared field lines build up and the current density is enhanced significantly, differs from that of the potential field. Figure 6 e shows the $171 \AA$ EUV images for the time period in Fig. 6a; these were acquired

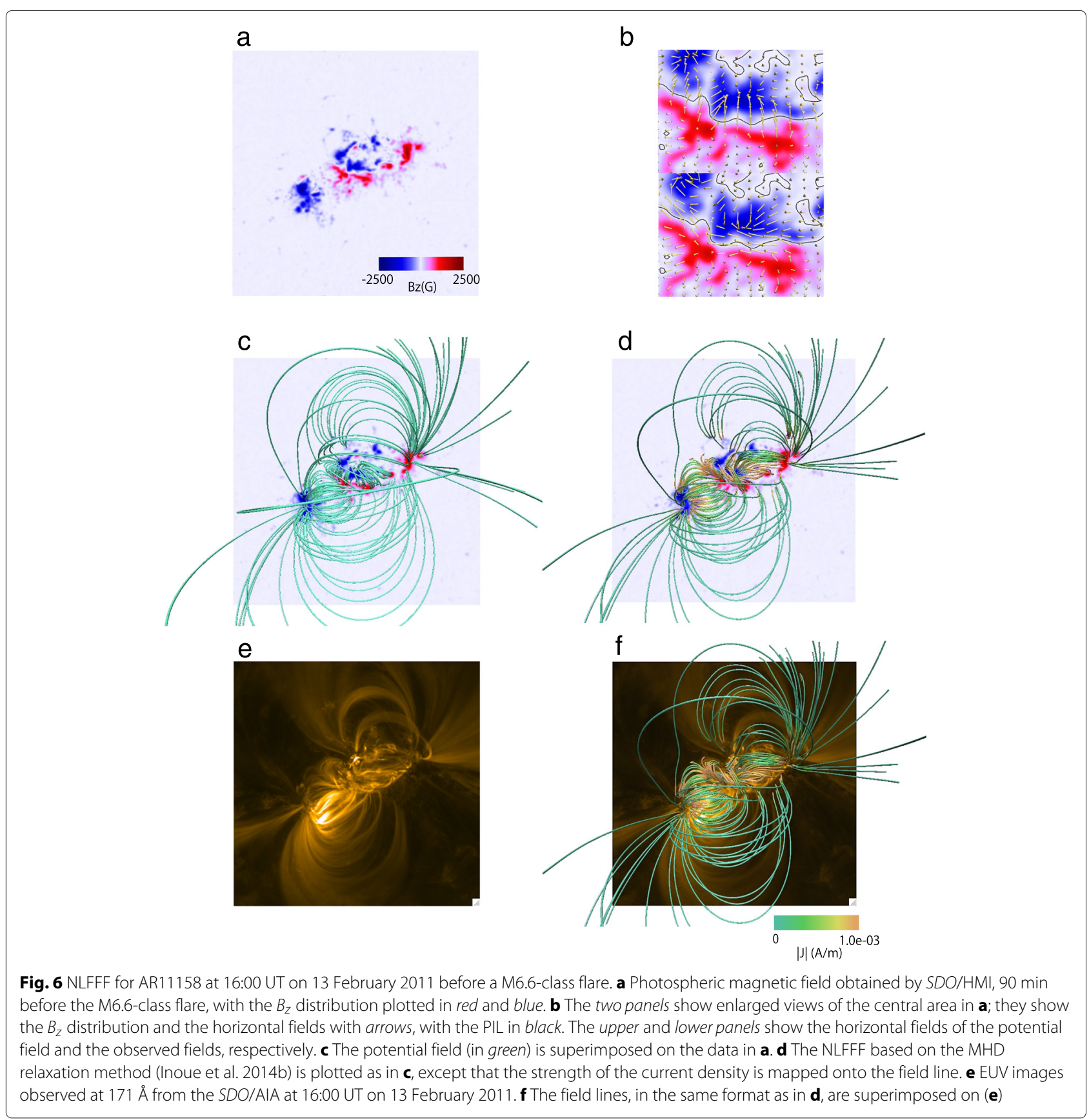


by an atmospheric imaging assembly (AIA; Lemen et al. (2012)) on board SDO. The same field lines as in Fig. 6d were superimposed on Fig. 6e. Because it can be clearly seen that most of the field lines roughly correspond to these obtained from the EUV image, the NLFFF appears to satisfactory reproduce the field lines in the observed EUV image.

\section{Stability analysis of the NLFFF}

Magnetic field stability is an important issue in the studies of solar eruptions. Unfortunately, the photospheric magnetic field and the EUV or X-ray images do not allow for a quantitative stability analysis. On the other hand, the NLFFF might allow a quantitative analysis if we are given the 3D space information. One of the possible instabilities that can drive an eruption under the zero-beta assumption is a current driven, and one type current-driven instability is kink instability (Ji et al. 2003), which is determined by the magnetic twist of the poloidal field generated by the current in the flux tube (Kruskal and Kulsrud 1958). The magnetic twist $\left(T_{n}\right)$ is related to the magnetic helicity; that is, the flux tube helicity is described by the following equation (Berger and Field 1984):

$$
H=\left(T_{n}+W_{r}\right) \Phi^{2},
$$

where $H$ is the magnetic helicity, $\Phi$ is the magnetic flux of the flux tube, and $W_{r}$ is the magnetic writhe corresponding to the helical structure of the field line axis. The magnetic twist $T_{n}$ indicates how much of the magnetic helicity is generated by the currents parallel to the flux tube (Berger and Prior 2006; Török et al. 2010); thus, $T_{n}$ can be written as

$$
T_{n}=\int \frac{d T_{n}}{d s} d l=\int \frac{J_{\|}}{4 \pi|\boldsymbol{B}|} d l
$$

where || indicates the component parallel to the field line, and the line integral $\int d l$ is taken along the magnetic field line of the flux tube. Using $J_{\|}=\boldsymbol{J} \cdot \boldsymbol{B} /|\boldsymbol{B}|$, Eq. (55) can be further rewritten as

$$
T_{n}=\frac{1}{4 \pi} \int \frac{\boldsymbol{J} \cdot \boldsymbol{B}}{|\boldsymbol{B}|^{2}} d l
$$

If the magnetic fields meet the force-free condition, the magnetic twist can be written as

$$
T_{n}=\frac{1}{4 \pi} \int \alpha d l=\frac{1}{4 \pi} \alpha L,
$$

where $\alpha$ is the force-free alpha, and $L$ is the length of the field line (Inoue et al. 2011; Inoue et al. 2012a). Inoue et al. (2012b) and Inoue et al. (2013) performed a stability analyses on the NLFFFs of AR10930 and AR11158, both of which produced X-class flares. Below, we describe the results of one of these twist analyses (for AR11158). AR 11158 produced an X2.2-class flare at 01:50 UT on 15 February 2011; it exhibited a quadruple field, as shown in
Fig. 7a. The NLFFF based on the MHD relaxation method is shown in Fig. 7b; strong twisted lines were formed in the central region. The twist $T_{n}$ was calculated for all field lines according to Eq. (56), and the result is shown in Fig. 7c. According to this result, most of the field lines were less than one turn, and none reached the critical twist of $T_{n}=1.75$, which is required for kink instability (Török et al. 2004). Therefore, it was concluded that the twisted lines prior to the X2.2-class flare produced by AR11158 would be stable with respect to kink instability. In another study, Jiang et al. (2014a) successfully reproduced a large twisted filament and checked its stability. It was reported that the twist did not reach the critical value required for kink instability. However, note that $T_{n}$ in Eq. (56) is the local twist of an infinitesimal flux tube; this is not the same as the global twist of a macroscopic flux rope. In addition, there is no guarantee that the theoretical criteria are directly applicable to the NLFFF. In order to more strictly confirm the stability, a numerical stability analysis (Kusano and Nishikawa 1996; Inoue and Kusano 2006) and an MHD simulation would be useful.

Torus instability (Kliem and Török (2006); tested against observations by Liu (2008)) is also important for driving the flux tube into the upper corona, e.g., for triggering a CME (Isenberg and Forbes 2007; Aulanier et al. 2010; Démoulin and Aulanier 2010; Kliem et al. 2014). This instability is induced by a broken force balanced against the hoop force (Chen 1989), due to the flux tube current and the magnetic field suppressing the flux tube. The decay index,

$$
n(z)=-\frac{z}{|\boldsymbol{B}|} \frac{\partial|\boldsymbol{B}|}{\partial z}
$$

is a convenient parameter (Kliem and Török 2006) because the location where this instability takes place is specified by $n=1.5$, which was already confirmed by several numerical studies (Török and Kliem 2007; Aulanier et al. 2010; Fan 2010). This stability analysis can be applied to the NLFFF analysis. For example, Guo et al. (2010) reconstructed the NLFFF using the optimization method (Wiegelmann 2004). In contrast to Inoue et al. (2011), they found strongly twisted lines over the critical twist of the kink instability and its writhe motion during the flare while a confined eruption was observed. They pointed out that even though the twisted lines in the NLFFF were not stable with respect to the kink instability, they were stable with respect to the torus instability, i.e., the flux tube remains within the magnetic field satisfying $n \leq 1.5$ during the eruption. Regarding the AR11158 studied by (Inoue et al. 2014a), the decay index at the twisted lines formed in the NLFFF cannot reach the critical value of the torus instability, as shown in Fig. 7d. Thus, the authors pointed out that the NLFFF was stable with respect to both torus instability and kink instability. On the other 
a

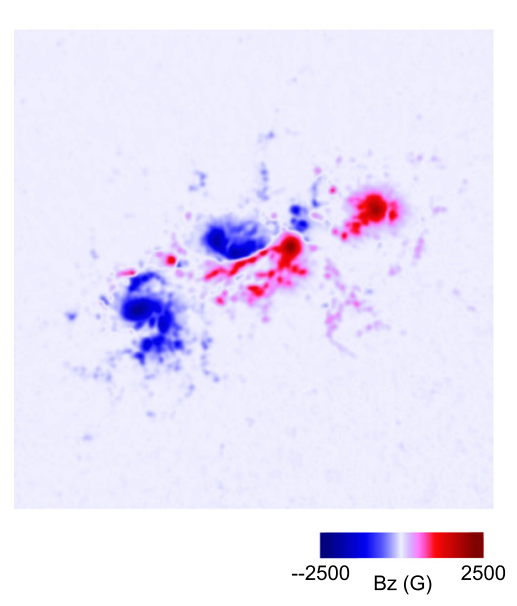

C

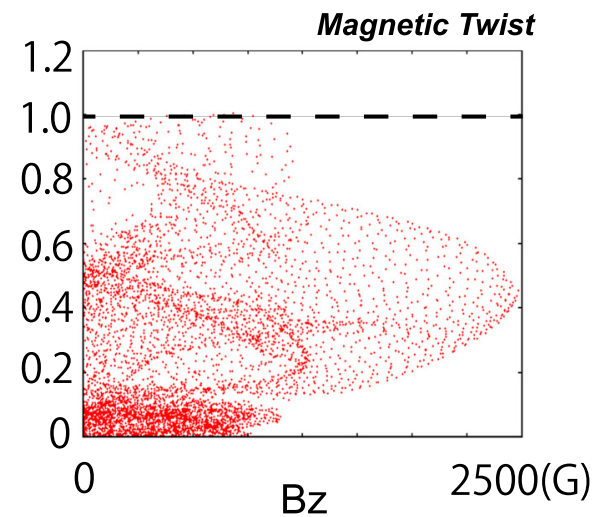

b

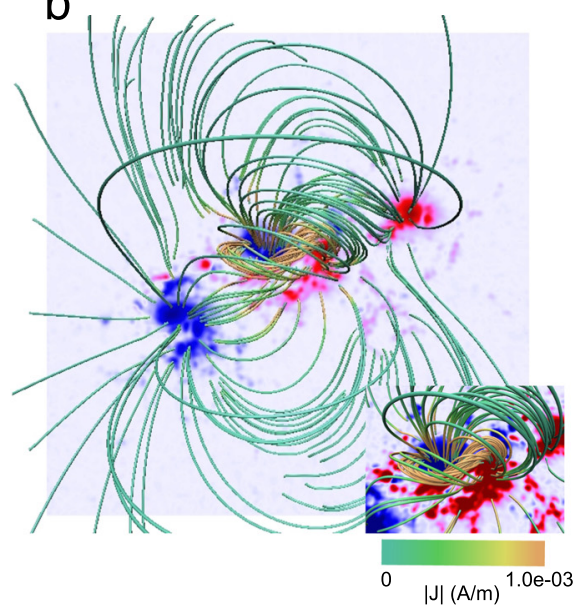

d

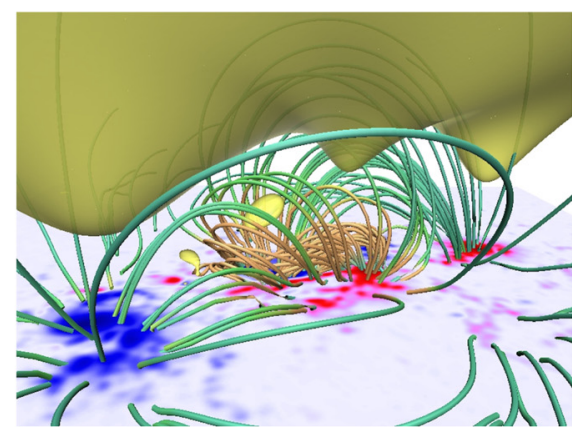

Fig. 7 NLFFF for AR1 1158 at 00:00 UT on 15 February 2011 before a X2.2-class flare. a The $B_{z}$ distribution of the photospheric magnetic field, approximately $2 \mathrm{~h}$ before the occurrence of the X2.2-class flare observed by SDO/HMI. b Magnetic field lines from the NLFFF are superimposed on $\mathbf{a}$ : the format of the field lines is the same as in Fig. $6 \mathrm{~d}$. The small inset corresponds to an enlarged view of the central area. c The magnetic twist distribution from Inoue et al. (2014a), where the vertical and horizontal axes are the twist and $B_{z}$, respectively. The dashed line corresponds to $T_{n}=$ 1.0. The image is copyright AAS and reproduced by permission. $\mathbf{d}$ The magnetic field lines are plotted together with the surface corresponding to the critical height of the torus instability

hand, for a different event, Jiang et al. (2014b) estimated the temporal evolution of the flux tube height obtained from the NLFFF in solar active region 11283, focusing on the X2.1-class flare that occurred at 22:20 UT on 6 September 2011. They found that the decay index at the flux rope axis reached the critical value for torus instability at the time at which the flare was generated, resulting in an instability-driven eruption.

As seen from these studies, the NLFFF enables us to quantitatively perform a stability analysis, which would be difficult to do based only on observations. Recently, highly accurate measurements of photospheric magnetic fields became available from two space satellites and ground observations; these have made the NLFFF a very useful tool for understanding the coronal magnetic field as well as for speculating on the onset and dynamics of solar flares.

\section{MHD simulations of the solar eruptions based on the} observational data

\section{Necessity of MHD simulations combined with the NLFFF}

Numerical modeling of the coronal magnetic field (potential field, LFFF, and NLFFF) successfully clarified many unknown issues with 3D magnetic fields that had not been revealed by observation. On the other hand, these models consider only the force-free equilibrium state, and they are thus not able to model dynamic states (in particular, energy-released processes) that occur during flare events, even though the buildup of energy occurs at a rate much slower than the Alfven time scale and thus can be handled by the NLFFF. MHD simulations can be used to reproduce such dynamic states.

The potential field does not strongly contribute to the magnetic field in the solar active region because there is no free energy available to induce dynamic behavior. 
For instance, Zuccarello et al. (2012) performed MHD simulations of solar eruptions, using the potential field as the initial condition. To obtain the solar eruption, the Poynting flux through the boundary was determined, and the authors provided the hypothetical shear and the convergence of the plasma on the solar surface. Consequently, the non-potential field was built up, and the sheared and converging motions helped to form the flux tube, resulting in an eruption (Figure 6 and Figure 8 in their paper). The hypothetical motions are important factors for building up the non-potential field, but these are much different from the observed ones. This means that there is a different process for the building up of energy, i.e., the magnetic field just prior to the onset of a flare deviates from the observed one. In contrast to this process, several studies inserted an analytical flux rope with a strong current and non-potentiality in a local area close to the PIL into the reconstructed potential field. Unfortunately, these flux tubes did not agree exactly with the observations, i.e., the boundary condition of the flux tube deviated greatly from the observations.

It might be possible to overcome the above problem by using MHD simulations with the NLFFF because the NLFFFs are constructed on the photospheric magnetic field, including the observed horizontal magnetic field on the solar surface. The motivations for using these simulations rather than the previous one are as follows: (i) It is likely that the artificial energy buildup process is not required by the existence of twisted motions because it already accounts for the observed twisting in the NLFFF. Although an additional process is required (discussed below) to create a new state that deviates from the NLFFF and produces eruptions, compared to the previous simulations, that process does not greatly deform the initial state. Therefore, MHD simulations can be performed under the photospheric magnetic field constraint. (ii) These simulations allow for the study of complex nonlinear dynamics, which could not be done previously. (iii) The results obtained from these simulations can be compared more exactly with observations, even indirect ones. Thus, these results contribute to confirming the reliability or to improving the MHD model. This field of study is emerging (Jiang et al. 2013), and only a few papers have yet been published. Below, we briefly discuss several of the pioneering studies.

MHD models of the solar eruptions, combined with the NLFFF Overview of the recent studies. Jiang et al. (2013) were the first to perform the MHD simulation using the NLFFF to reproduce the X2.1-class flare in solar active region 11283. Their NLFFF, which was reconstructed by using the MHD relaxation method constructed in the modern MHD scheme (Feng et al. 2010), successfully captured the sigmoid structure of the magnetic field observed before the flare and demonstrated that the eruption was driven by the torus instability (Fig. 8a). An important advantage of this study seems to be that the same algorithm was used in both the NLFFF and MHD simulations. Kliem et al. (2013) also studied this eruption by setting the NLFFF as the initial condition of their MHD simulation (Fig. 8b). The NLFFF was reconstructed using the magnetic field observed on 8 April 2010, using the flux rope insertion and the magnetofrictional method. The NLFFF of this active region was thoroughly studied by $\mathrm{Su}$ et al. (2011). Kliem et al. (2013) found a critical value of the axial flux in the flux rope determined the stability. They reported that the criteria for the onset of a flare is that the axial flux be in the range of $5 \times 10^{20}$ to $6 \times 10^{20} \mathrm{Mx}$; in this case, the decay index is in the range of 1.3 to 1.8 . For this eruption, the simulation results were in good agreement with some of the observations, such as those during the initial rising phase leading to the eruption. Amari et al. (2014) also successfully demonstrated a flux tube eruption in their MHD simulations, as shown in Fig. 8c. The flux tube was reconstructed by using the Grad-Rubin type method (Amari and Aly 2010) combined with the photospheric magnetic field observed by the Hinode solar optical telescope (SOT; Tsuneta et al. (2008)) $6 \mathrm{~h}$ before the X3.4-class flare in AR10930 at 02:40 UT on 13 December 2006. The authors found that $6 \mathrm{~h}$ before the flare, the NLFFF was destabilized with flux cancellation, the gas motion in characteristic of a sunspot moat flow or photospheric turbulent diffusion, and this resulted in the eruption. On the other hand, 2 days before the flare, the NLFFF predicted no eruption for the same situation. The authors pointed out the importance of the formation of a significantly large flux tube and the moving out from equilibrium.

MHD modeling of the solar eruption on 15 February 2011. Inoue et al. (2014a) and Inoue et al. (2015) studied the magnetic field dynamics during the X2.2-class flare produced by solar active region 11158 on 15 February 2011 (Schrijver et al. 2011; Janvier et al. 2014; Yang et al. 2014), by using MHD simulations combined with the NLFFF. Figure $7 \mathrm{~b}$ shows the NLFFF structure approximately $2 \mathrm{~h}$ before the X2.2-class flare on 15 February 2011; note that strongly sheared magnetic fields lines are clearly visible at the PIL of the central sunspot. The stability analysis was discussed in a previous section. Based on these results, the NLFFF was quite stable, which implies that an additional process is required to drive the twisted lines. For instance, in a detailed data analysis, (Bamba et al. 2013) observed an increase in the small flux emerging at the PIL before the flare, and they suggested 


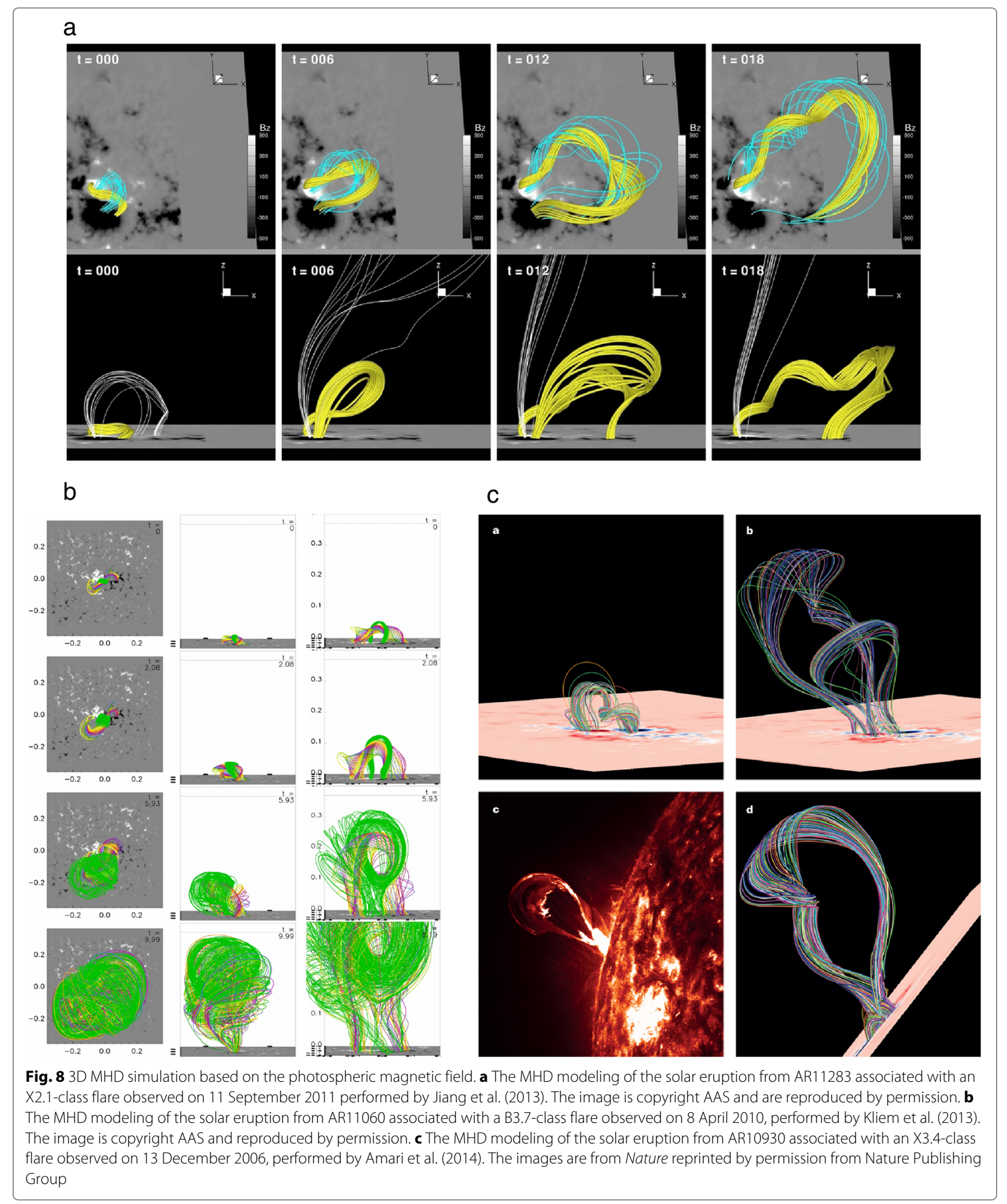

that this could destroy the stable magnetic field, as in the scenario described by Kusano et al. (2012).

The dynamics were investigated in the zero-beta MHD approximation, i.e., the density, pressure, and gravity were neglected. In such a situation, although the thermodynamics during the flare cannot be investigated, the magnetic field dynamics can be considered (Inoue et al. 2014a). This is the case because, during the flare, the 
magnetic energy converts into kinetic energy and thermal energy, which are the main factors for the energy storeand-release process in the solar corona. Therefore, in the early phase of a solar eruption that is not strongly compressible, zero-beta plasma is a good approximation, as demonstrated by Inoue and Kusano (2006). An advantage of this approximation is that it can neglect the sound waves, which often highly influence the rarefied CFL condition.

As discussed in the previous section, the NLFFF reconstructed $2 \mathrm{~h}$ before the X2.2-class flare shows a stable equilibrium state, and the dramatic dynamics that appear in observations are not evident. Therefore, some additional process is required to break the stable equilibrium. Here, Inoue et al. (2014a) and Inoue et al. (2015) introduced an anomalous resistivity imposed on the strong current region, and the MHD relaxation was performed by using the NLFFF as the initial condition where the velocity adjustment defined in Eq. (50) was removed. We would expect the anomalous resistivity to induce reconnection in the region of strong current density (Yokoyama and Shibata 2001) and to produce long twisted lines in the NLFFF. After an additional iteration, since there is no guarantee that this new state can remain in equilibrium, a newly created flux tube might escape from the solar surface, as was shown in Amari et al. (2014). The anomalous resistivity was

$$
\eta= \begin{cases}\eta_{0} & J<j_{c}, \\ \eta_{0}+\eta_{2}\left(\frac{J-j_{c}}{j_{c}}\right)^{2} & J>j_{c},\end{cases}
$$

where $\eta_{0}$ is the background resistivity and $j_{c}$ is the threshold current necessary to excite the second term in Eq. (59) (Yokoyama and Shibata 1994). In this study, $\eta_{0}=1.0 \times$ $10^{-5}, \eta_{2}=1.0 \times 10^{-4}$, and $J_{c}=30$. It can initiate and enhance the reconnection in the strong current region when the current is greater than the critical value, $J_{c}$. This value depends on the normalized value of the coronal magnetic field defined in each study.

Figure 9a shows two bundles of the twisted lines formed in the NLFFF; a strong current region was formed and sandwiched by these bundles. The side view is shown in Fig. 9b. We expect that reconnection takes place between the two bundles of the twisted lines, and long, strongly twisted lines are formed, which might break the equilibrium. After additional iterations with the anomalous

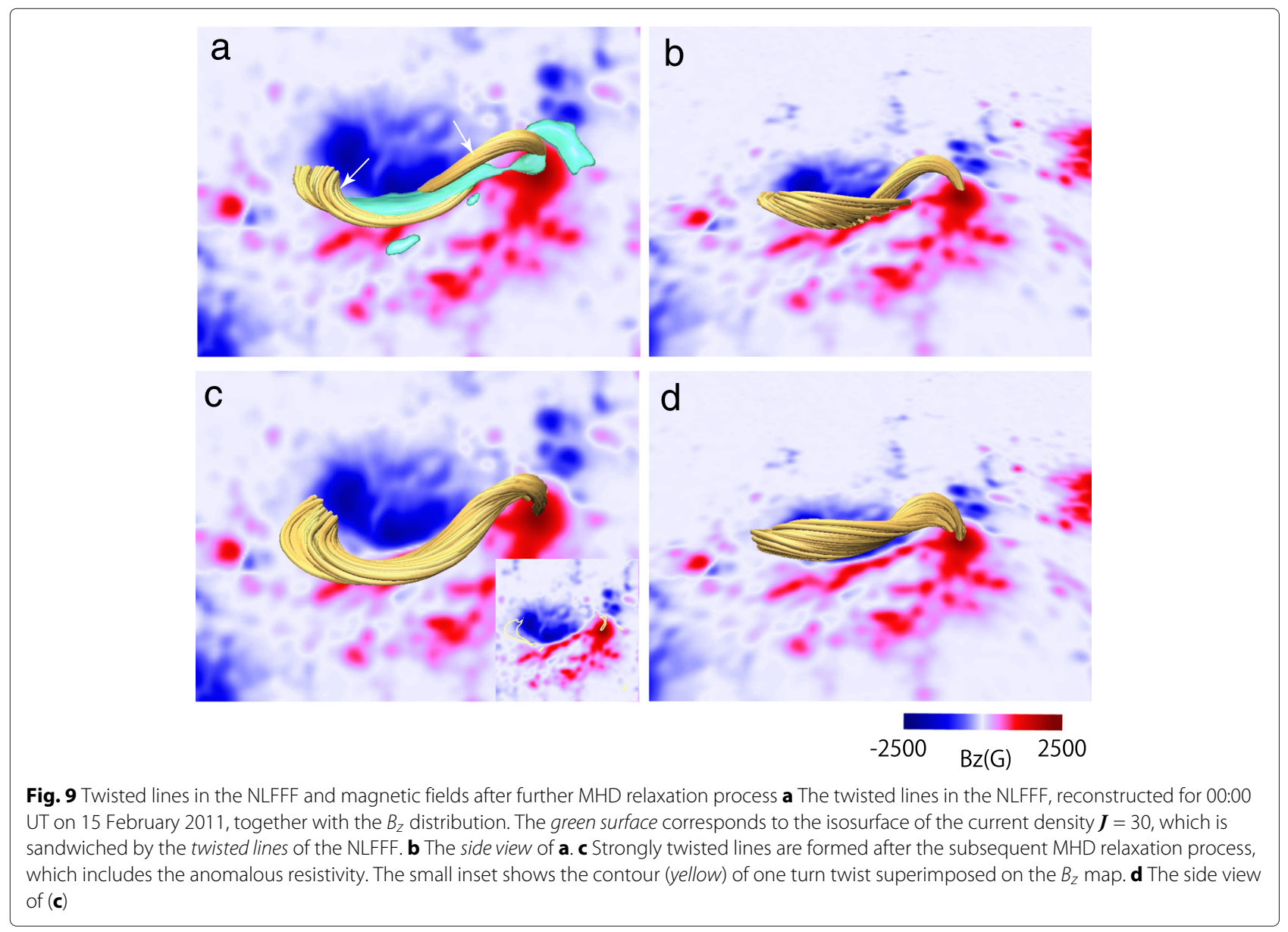



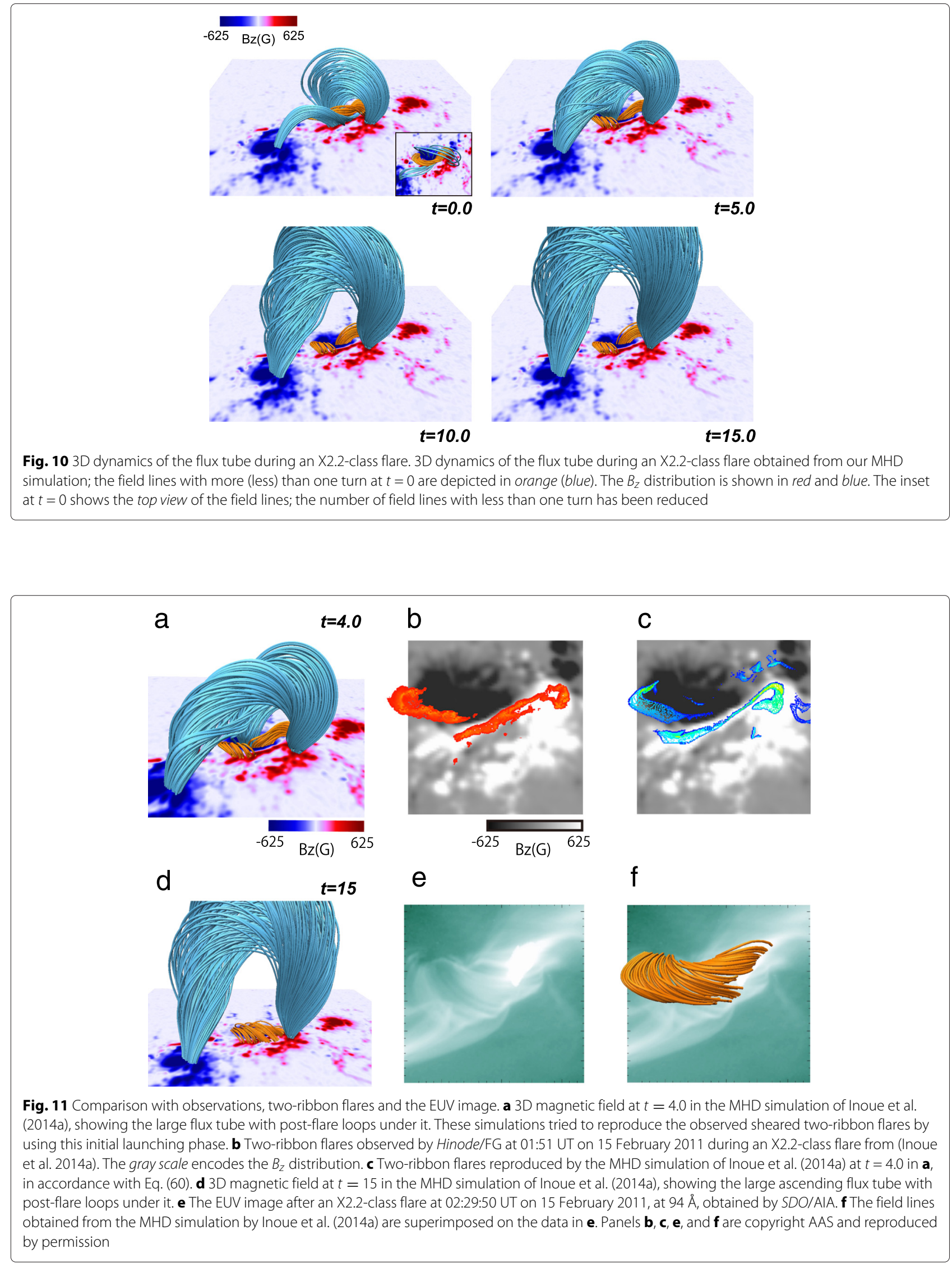
resistivity, the single long bundle of strongly twisted lines shown in Fig. 9c, d was produced by the reconnection between the twisted lines formed in the NLFFF (shown in Fig. 9a); which is reminiscent of the tether-cutting reconnection shown in Fig. 3e. The small inset shown in Fig. 9c shows the contour of one twist superimposed on the $B_{z}$ map, where the footpoints for the part of the selected field lines plotted in Fig. 9c, d are anchored inside this contour. Note that there is no guarantee that this new state can remain in equilibrium because a flux tube composed of strongly twisted lines can escape from the solar surface (Amari et al. 2000; Kusano et al. 2012; Kliem et al. 2013).

Next, an MHD simulation was executed using this new state, as shown in Fig. 9c; note that at the boundary, all components of the velocity are fixed to zero, and the normal component of $\boldsymbol{B}$ is fixed, while the horizontal one may vary, i.e., it is determined by the induction equation according to the dynamics. Consequently, as shown in Fig. 10, the equilibrium was broken, and a larger flux tube was formed and launched into the upper corona. Note that, in this process, the strongly twisted lines (in orange) that were formed during the initial state do not extend directly into the upper corona.
Rather, they reconnect with the ambient field lines (in blue) that convert into the large flux tube. Interestingly, the strongly twisted lines in the initial state appear to convert into the post-flare loops often observed after a flare.

These simulation results were compared with observations. The authors first confirmed that their simulation captures the shape of the two-ribbon flares. Following the CSHKP model, two-ribbon flares are generally considered to be due to the distribution of the footpoints of the reconnected field lines. Therefore, those could be reproduced to trace the reconnected field lines during a simulation. To achieve this, the authors traced the reconnected field lines by using the following equation:

$$
\delta\left(x_{0}, t_{n}\right)=\left|x_{1}\left(x_{0}, t_{n+1}\right)-x_{1}\left(x_{0}, t_{n}\right)\right|,
$$

where $t_{n+1}$ is the next time step after $t_{n}$, and $\boldsymbol{x}_{1}\left(\boldsymbol{x}_{0}, t_{n}\right)$ is the location of one footpoint of each field line at time $t_{n}$, which is traced from another footpoint at $\boldsymbol{x}_{0}$. Eventually, we calculate

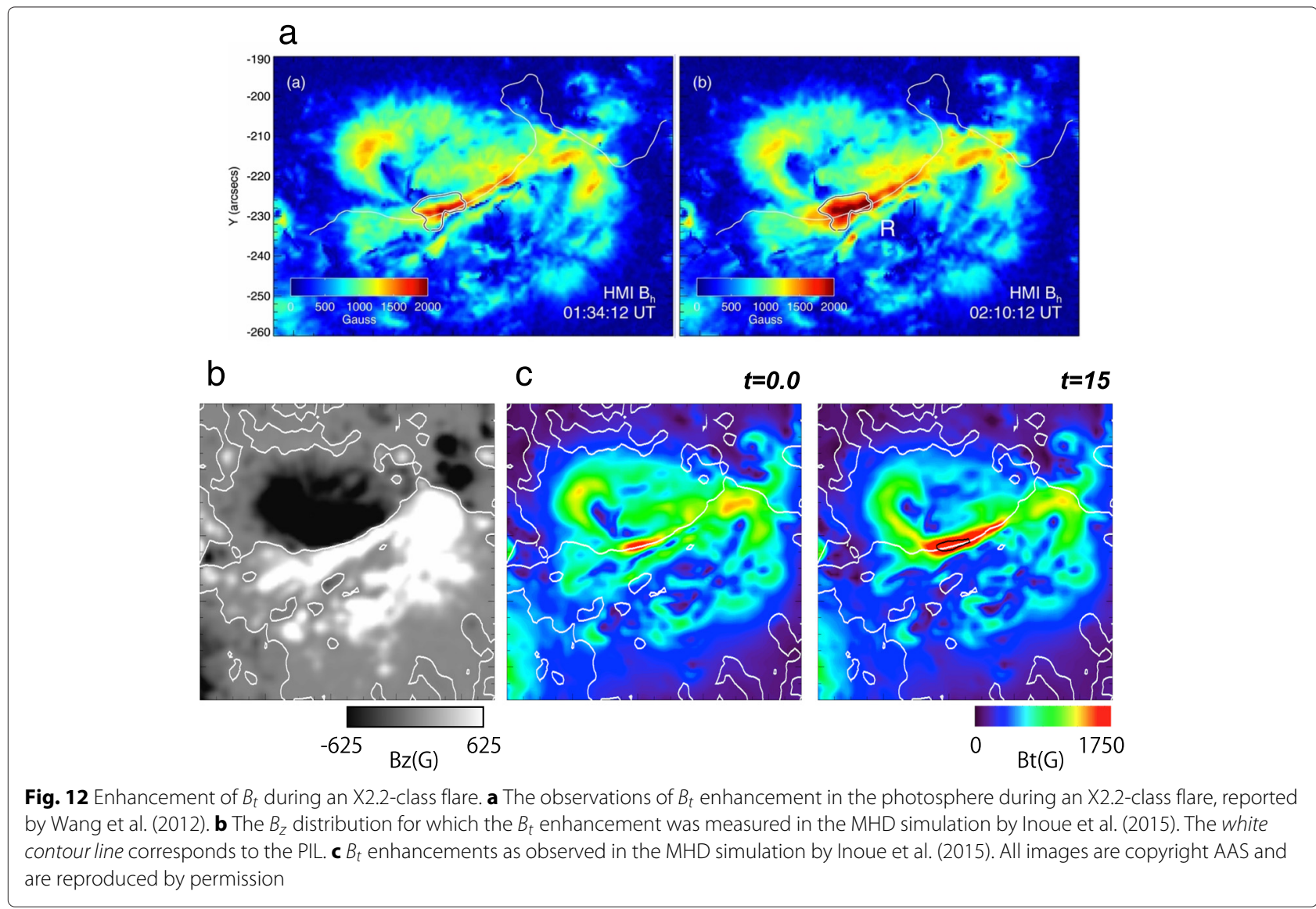




$$
\Delta\left(\boldsymbol{x}_{0}, t\right)=\int_{0}^{t} \delta\left(\boldsymbol{x}_{0}, t_{n}\right) d t_{n}
$$

where $\Delta\left(x_{0}, t\right)$ is a location where the length of a field lines is changed, meaning that the enhanced region corresponds to one in which there was a dramatic reconnection in the twisted lines. Figure 11a shows a $3 \mathrm{D}$ view of the field lines at $t=4.0$, when the large flux tube has been formed during the initial launching phase. We first confirmed that the sheared two-ribbon profiles observed initially were reproduced in our simulation. Figure $11 \mathrm{~b}$ shows the two-ribbon flares during the X2.2-class flare, observed by Hinode/SOT, at 01:50 UT, corresponding to the initial phase of the flare. Figure 11c shows the numerically calculated two-ribbon flares, following Eq. (60), at $t=4$, reproduced in this simulation where $\Delta$ is chosen from the region in which $T_{n}>0.3$. The shape of the numerically calculated two-ribbon flares matches the observed one.

These simulation results were further compared with the EUV image data obtained from SDO/AIA. Figure 11d shows the $3 \mathrm{D}$ magnetic structure at $t=15$, clearly revealing the post-flare loops above which the large eruptive flux tube is ascending. We confirmed that the post-flare loops can capture the field lines in the EUV image, using simulation data at $t=15$. Figures $11 \mathrm{e}$ shows the EUV image observed after the flare by $94 \AA$ of SDO/AIA, and Fig. $11 \mathrm{f}$ shows the field lines at $t=10$ superimposed on the EUV image. The field lines observed in the EUV image were successfully captured.

Finally, enhancement of the horizontal magnetic field $B_{t}$ was discussed by Inoue et al. (2015). As shown in Fig. 12a, Wang et al. (2012) found a rapid enhancement of the horizontal field on the PIL in the photosphere, and they suggested that this was due to the

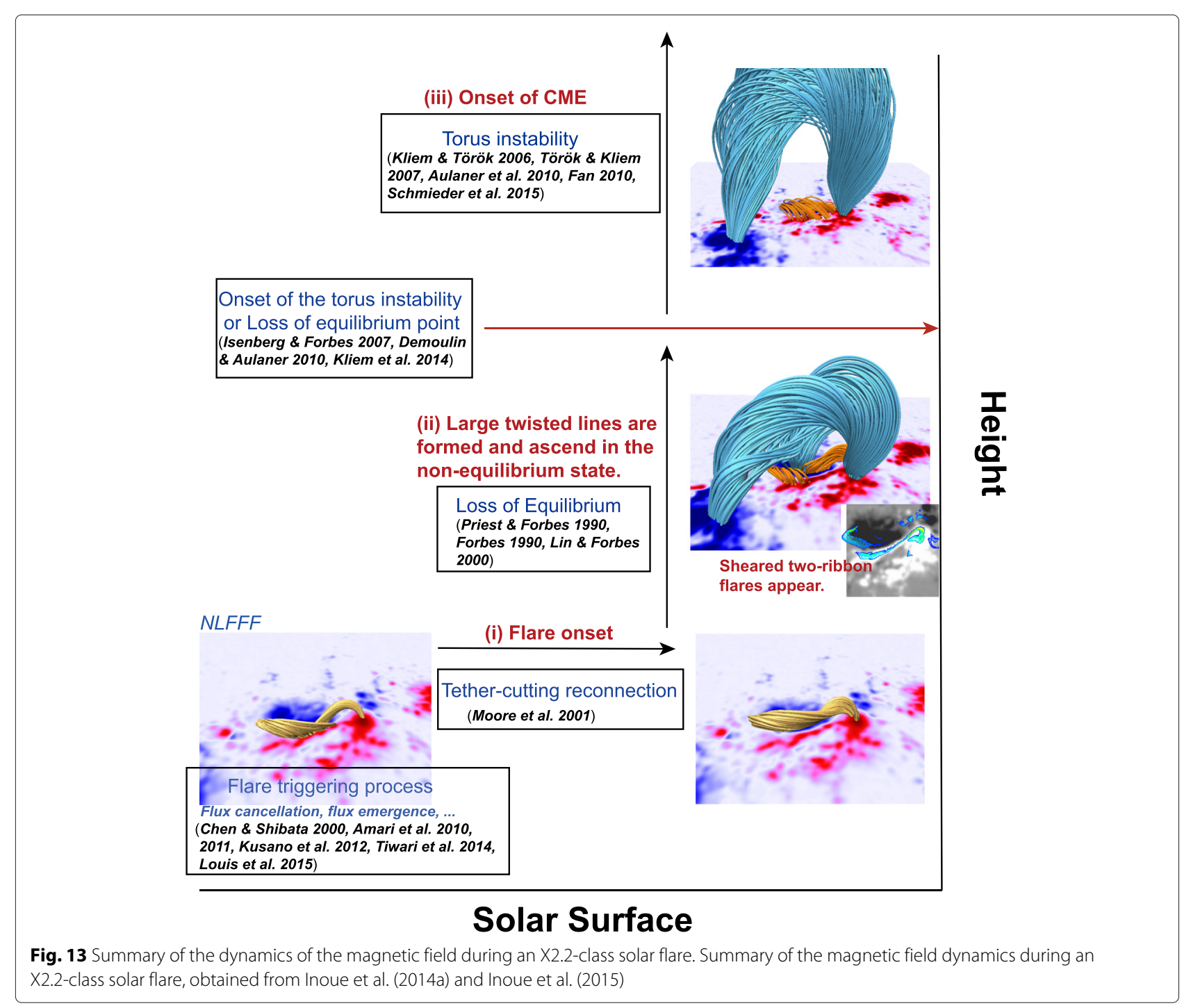


reconnection. The simulation of Inoue et al. (2015) also indicated this enhancement, and the result is shown in Fig. 12c calculated for the area shown in Fig. 12b. It was pointed out that the post-flare loops can be observed even during an early phase in which the horizontal fields are enhanced, and the new post-flare loops are subsequently produced through the above reconnection. Consequently, it was suggested that this enhancement is due to the accumulation of post-flare loops suppressing the pre-existing loops. Therefore, this enhancement would be strongly related to the reconnection. However, since this simulation was performed in the zero-beta MHD, to support this conclusion, it is necessary to have a more detailed analysis and discussion under more realistic assumptions, including high- $\beta$ regimes corresponding to the chromosphere and the photosphere.

A summary of the dynamics is shown in Fig. 13. (i) The NLFFF is quite stable for the current-driven ideal MHD instability, so it is necessary to have a trigger process (here, the tether-cutting reconnection) to break the equilibrium. (ii) The tether-cutting reconnection creates strongly twisted lines in the NLFFF. Consequently, it breaks the equilibrium and reconnects with the ambient field lines. The result is that a large flux tube is formed as it ascends. (iii) Eventually, the flux tube will grow into a CME if the threshold of the torus instability is exceeded or equilibrium is lost.

\section{Conclusions}

The solar physics satellites Hinode and SDO, together with modern ground-based telescopes, provide photospheric magnetic field data with unprecedented accuracy. This enables us to reconstruct the 3D coronal magnetic field with high accuracy, such that it includes the potential field from the normal component not only of the photospheric magnetic field but also of the NLFFF, which contains both the normal and the horizontal magnetic fields. Because the NLFFF is reconstructed to include information about the horizontal magnetic fields at the photosphere, it can yield a 3D magnetic field close to that observed in the active regions instead of the one similar to that of the potential field, and it can show the accumulation of free magnetic energy and helicity that is required to produce a flare. In addition, the force-free $\alpha$ is given as a function of space, and so it is not an LFFF approximation. Therefore, the NLFFF can yield the magnetic configuration both before and after the flare, and several papers have reported various important physical quantities obtained from the NLFFF, including the free magnetic energy (Sun et al. 2012; Jiang et al. 2014b), the magnetic helicity (Thalmann et al. 2011; Valori et al. 2012; Pevtsov et al. 2014), and the magnetic twist and topology (Inoue et al. 2011; Guo et al. 2013; Inoue et al. 2013; Zhao et al. 2014). These quantities quantify the NLFFF stability, which cannot be obtained from observations.

On the other hand, there is a problem in the NLFFF itself. Using the same format as in Fig. 5d, Fig. 14 shows the distribution of the force-free $\alpha$ measured at both footpoints of each field line for the NLFFF in Fig. $7 \mathrm{~b}$ and the temporal evolution of $\int|\nabla \cdot \boldsymbol{B}|^{2} \mathrm{dV}$ during the iteration of the NLFFF. Although the value of $\int|\nabla \cdot \boldsymbol{B}|^{2} \mathrm{dV}$ is reduced to fourth order, the distribution of the force-free $\alpha$ is scattered. Therefore, an unexpected physical element, the residual force, remains; this is inevitably produced near the boundary in the NLFFF, due to the contradiction between the boundary and the inner domain. In addition, it should be noted that the coronal magnetic fields cannot be correctly reproduced only by the NLFFF. Peter et al. (2015) pointed out several limitations on the free energy and accumulated currents. Furthermore, reconstruction of the geometry of bright loops requires
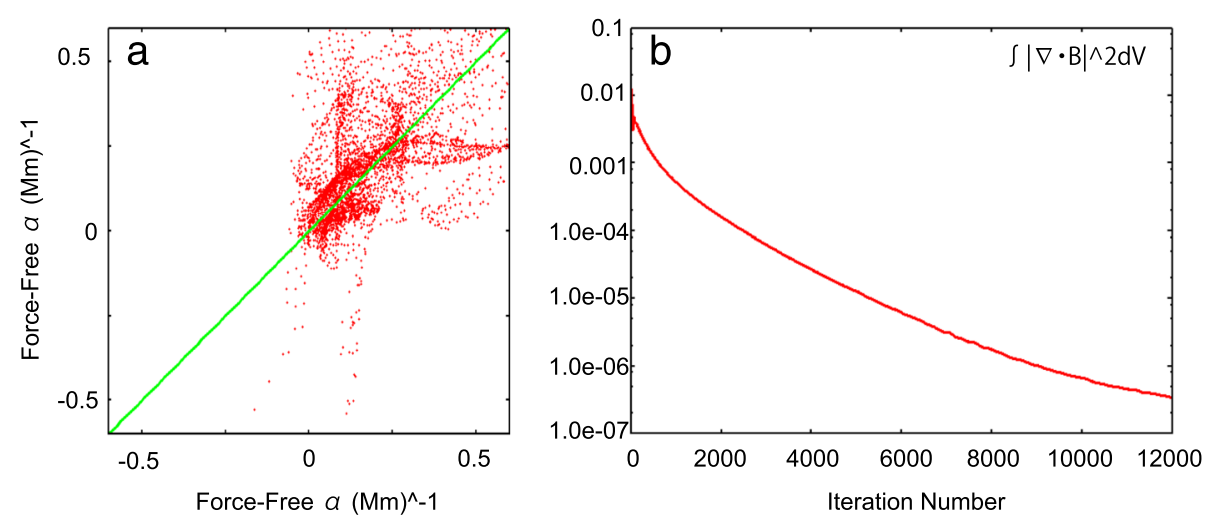

Fig. 14 Force-freeness of the NLFFF. a Distribution of the force-free $\alpha$ measured at both footpoints of each magnetic field line for the NLFFF in Fig. 7b, that is from Inoue et al. (2014a). The image is copyright AAS and reproduced by permission. $\mathbf{b}$ The temporal evolution of $\int \nabla \cdot \boldsymbol{B} d V$ during the iteration in which the NLFFF is attained 
methods more advanced than the NLFFF (Aschwanden et al. 2014; Malanushenko et al. 2014). Therefore, a model more advanced than the NLFFF is required to construct the equilibrium state with high accuracy and overcome these limitations.

Although the NLFFF yields the 3D properties of the magnetic field, this method does not reveal the dynamics of the solar flares. To determine the dynamics in a realistic situation, NLFFF results have been used as initial conditions for MHD simulations (Jiang et al. 2013; Kliem et al. 2013; Inoue et al. 2014a; Amari et al. 2014; Inoue et al. 2015). Because these simulations were constrained by the photospheric magnetic field, there are large artificial processes causing the buildup of energy; these likely yield twist and sheared motions, which were not assumed. Important and realistic physical processes are also being revealed, including the critical value for the flux of the flux tube for an eruption (Kliem et al. 2013) or the formation of a large flux tube producing a CME (Inoue et al. 2014a; 2015). Furthermore, the reliability of these simulations can be confirmed because they can be more precisely compared with the observations likely by Inoue et al. (2014a) and Inoue et al. (2015), in contrast to previous simulations that described hypothetical situations. Note that several can be indirectly compared, e.g., the two-ribbon flares discussed in this study. In order to provide a strict confirmation, however, a direct comparison is required (e.g., (Mikić et al. 2013)).

Some problems and questions related to these simulations still remain to be answered. For instance, as discussed above, the reconstructed field does not completely achieve a force-free state, and so the residual force must be treated carefully. If these residual forces are sufficiently strong, they may affect the magnetic field dynamics, and the interpretation of the dynamics becomes difficult. In addition to this, as Inoue et al. (2015) pointed out, the magnetic twist accumulated in the NLFFF might be gradually reduced throughout the numerical diffusion and also on the solar surface because the NLFFF returns to a lower energy level without retaining the observed horizontal magnetic fields. Furthermore, it is important to account for the observed process that triggers the solar flares in order to understand the conversion of the stable magnetic field into a dynamic one. Recently, the triggering was observed by using state-of-art data (e.g., Green et al. (2011); Bamba et al. (2013); Louis et al. (2015)). These data must be incorporated into simulations. Although most simulations start from an NLFFF that is already composed of twisted and sheared field lines, some studies attempted to recover the processes leading from the buildup to the release of energy; in this data-driven simulation, the coronal magnetic field was driven by the time-dependent photospheric magnetic field e.g., Cheung and De Rosa (2012).
Work in this direction is currently underway, and this will be extended in the future.

With advanced computational resources now more readily available, more-refined 3D numerical MHD models of solar eruptions are being developed and improved. Recently, techniques combining simulations with highly resolved temporal and spatial data from state-of-theart solar satellites have been developed, and these have yielded some preliminary results. In the future, it is likely to be necessary to further develop simulations of solar flares in order to more closely correspond to these observations.

\section{Competing interests}

The author declares that he has no competing interests.

\section{Acknowledgements}

We thank the Japan Geoscience Union (JPGU) for inviting us to the JPGU 2014 meeting. We are grateful to the referees for providing many constructive comments and for suggesting ways in which to improve this paper. We are grateful to one of the science editors, Dr. Tsutomu Nagatsuma, for encouraging us, and to the MPS members for useful discussions. We offer special thanks to Dr. Takahiro Miyoshi and Dr. Vinay Shankar Pandey for checking a part of this paper. S. I. thanks the Alexander Von Humboldt Foundation for supporting our work and for providing a precious opportunity to work in Germany. This work was also supported by JSPS KAKENHI Grant Number $15 \mathrm{H} 05814$ (PI: K. Kusano). The computational work was carried out within the computational joint research program at the Institute for Space-Earth Environmental Research, Nagoya University. The computer simulation was performed on the Fujitsu PRIMERGY CX400 system of the Information Technology Center, Nagoya University. Visualization was performed by VAPOR (Clyne and Rast 2005; Clyne et al. 2007). Finally, S. I. sincerely thanks Professor Kanya Kusano for providing an opportunity to engage in this work and for many constructive comments and discussions.

Received: 15 December 2014 Accepted: 28 March 2016

Published online: 04 July 2016

\section{References}

Altschuler MD, Newkirk G (1969) Magnetic fields and the structure of the solar corona. I: Methods of calculating coronal fields. Solar Phys 9:131-149. doi:10.1007/BF00145734

Amari T, Aly J-J (2010) Observational constraints on well-posed reconstruction methods and the optimization-Grad-Rubin method. Astronomy Astrophys 522:A52. doi:10.1051/0004-6361/200913058

Amari T, Aly J-J, Luciani J-F, Mikic Z, Linker J (2011) Coronal mass ejection initiation by converging photospheric flows: toward a realistic model. Astrophys J Lett 742:L27-L32. doi:10.1088/2041-8205/742/2/L27

Amari T, Aly J-J, Mikic Z, Linker J (2010) Coronal mass ejection initiation: on the nature of the flux cancellation model. Astrophys J Lett 717:L26-L30. doi:10.1088/2041-8205/717/1/L26

Amari T, Boulmezaoud TZ, Aly JJ (2006) Well posed reconstruction of the solar coronal magnetic field. Astronomy Astrophys 446:691-705. doi:10.1051/0004-6361:20054076

Amari T, Canou A, J-Aly J (2014) Characterizing and predicting the magnetic environment leading to solar eruptions. Nature 514:465-469

Amari T, Luciani JF, Aly JJ, Mikic Z, Linker J (2003a) Coronal mass ejection: initiation, magnetic helicity, and flux ropes. I. Boundary motion-driven evolution. Astrophys J 585:1073-1086. doi:10.1086/345501

Amari T, Luciani JF, Aly JJ, Mikic Z, Linker J (2003b) Coronal mass ejection: initiation, magnetic helicity, and flux ropes. II. Turbulent diffusion-driven evolution. Astrophys J 595:1231-1250. doi:10.1086/377444

Amari T, Luciani JF, Mikic Z, Linker J (2000) A twisted flux rope model for coronal mass ejections and two-ribbon flares. Astrophys J Lett 529:L49-L52. doi:10.1086/312444

An JM, Magara T (2013) Stability and dynamics of a flux rope formed via flux emergence into the solar atmosphere. Astrophys J 773:21. doi:10.1088/0004-637X/773/1/21 
Antiochos SK, DeVore CR, Klimchuk JA (1999) A model for solar coronal mass ejections. Astrophys J 510:485-493. doi:10.1086/306563

Archontis V, Hood AW, Tsinganos K (2014) Recurrent explosive eruptions and the "sigmoid-to-arcade" transformation in the Sun driven by dynamical magnetic flux emergence. Astrophys J Lett 786:L21. doi:10.1088/2041-8205/786/2/L21

Asai A, Ishii TT, Kurokawa H, Yokoyama T, Shimojo M (2003) Evolution of conjugate footpoints inside flare ribbons during a great two-ribbon flare on 2001 April 10. Astrophys J 586:624-629. doi:10.1086/367694

Aschwanden MJ, Sun X, Liu Y (2014) The Magnetic field of active region 11158 during the 2011 February 12-17 flares: differences between photospheric extrapolation and coronal forward-fitting methods. Astrophys J 785:34. doi:10.1088/0004-637X/785/1/34

Aulanier G, Janvier M, Schmieder B (2012) The standard flare model in three dimensions. I. Strong-to-weak shear transition in post-flare loops. Astron Astrophys 543:AA110. doi:10.1051/0004-6361/201219311

Aulanier G, Török T, Démoulin P, DeLuca EE (2010) Formation of torus-unstable flux ropes and electric currents in erupting sigmoids. Astrophys J 708:314-333. doi:10.1088/0004-637X/708/1/314

Bamba Y, Kusano K, Yamamoto TT, Okamoto TJ (2013) Study on the triggering process of solar flares based on Hinode/SOT observations. Astrophys J 778:48. doi:10.1088/0004-637X/778/1/48

Benz AO (2008) Flare observations. Liv Rev Sol Phys 5:1. doi:10.1292/Irsp-2008-1

Berger MA, Field GB (1984) The topological properties of magnetic helicity. J Flu Mec 147:133-148. doi:10.1017/S0022112084002019

Berger MA, Prior C (2006) The writhe of open and closed curves. J Phys A: Math General 39:8321-8348. doi:10.1088/0305-4470/39/26/005

Bobra MG, van Ballegooijen AA, DeLuca EE (2008) Modeling nonpotential magnetic fields in solar active regions. Astrophys J 672:1209-1220. doi:10.1086/523927

Brackbill JU, Barnes DC (1980) The effect of nonzero product of magnetic gradient and $B$ on the numerical solution of the magnetohydrodynamic equations. J Comp Phys 35:426-430. doi:10.1016/0021-9991 (80)90079-0

Canou A, Amari T (2010) A twisted flux rope as the magnetic structure of a filament in active region 10953 observed by Hinode. Astrophys J 715:1566-1574. doi:10.1088/0004-637X/715/2/1566

Carmichael H (1964) A process for flares. In: AAS/NASA Symposium on physics of solar flares, NASA SP-50. NASA, Washington, DC. pp 451-456

Carrington RC (1859) Description of a singular appearance seen in the sun on September 1, 1859. MNRAS 20:13-15

Chen J (1989) Effects of toroidal forces in current loops embedded in a background plasma. Astrophys J 338:453-470. doi:1989ApJ...338..453C

Chen PF (2011) Coronal mass ejections: Models and their observational basis. Living Rev Solar Phys 8:1. doi:10.12942//rsp-2011-1

Chen PF, Shibata K (2000) An emerging flux trigger mechanism for coronal mass ejections. Astrophys J 545:524-531. doi:10.1086/317803

Cheng X, Zhang J, Ding MD, Liu Y, Poomvises W (2013) The driver of coronal mass ejections in the low corona: a flux rope. Astrophys J 763:43. doi:10.1088/0004-637X/763/1/43

Cheung MCM, DeRosa ML (2012) A method for data-driven simulations of evolving solar active regions. Astrophys J 757:147. doi:10.1088/0004-637X/757/2/147

Chiu YT, Hilton HH (1977) Exact Green's function method of solar force-free magnetic-field computations with constant alpha. I. Theory and basic test cases. Astrophys J 212:873-885. doi:10.1086/155111

Clyne J, Mininni P, Norton A, Rast M (2007) Interactive desktop analysis of high resolution simulations: application to turbulent plume dynamics and current sheet formation. New J Phys 9:301. doi:10.1088/1367-2630/9/8/301

Clyne J, Rast MM (2005) A prototype discovery environment for analyzing and visualizing terascale turbulent fluid flow simulations. Astrophysical and Planetary Sciences Faculty Contributions 5669:284-294. Paper 22. Proceedings of SPIE-IS andT Electronic Imaging. doi:10.1117/12.586032

Dedner A, Kemm F, Kröner D, Munz CD, Schnitzer T, Wesenberg M (2002) Hyperbolic divergence cleaning for the MHD equations. J Comput Phys 175:645-673. doi:10.1006/jcph.2001.6961

Démoulin P, Aulanier G (2010) Criteria for flux rope eruption: non-equilibrium versus torus instability. Astrophys J 718:1388-1399. doi:10.1088/0004-637X/718/2/1388

De Rosa ML, Schrijver CJ, Barnes G (2009) A critical assessment of nonlinear force-free field modeling of the solar corona for active region 10953. Astrophys J 696:1780-1791. doi:10.1088/0004-637X/696/2/1780
Evans CR, Hawley JF (1988) Simulation of magnetohydrodynamic flows: a constrained transport method. Astrophys J 332:659-677. doi:10.1086/166684

Fan Y (2005) Coronal mass ejections as loss of confinement of kinked magnetic flux ropes. Astrophys J 630:543-551. doi:10.1086/431733

Fan Y (2010) On the eruption of coronal flux ropes. Astrophys J 719:728-736. doi:10.1088/0004-637X/719/1/728

Feng X, Yang L, Xiang C, ST Wu, Zhou Y, Zhong DK (2010) Three-dimensional solar WIND modeling from the sun to the earth by a SIP-CESE MHD mode with a six-component grid. Astrophys J 723:300-319. doi:10.1088/0004-637X/723/1/300

Feynman J, Martin SF (1995) The initiation of coronal mass ejections by newly emerging magnetic flux. J Geophys Res 100:3355-3367. doi:10.1029/94JA02591

Forbes TG (1990) Numerical simulation of a catastrophe model for coronal mass ejections. J Goephys Res 95:11,919-11,931. doi:10.1029/JA095iA08p11919

Forbes TG (2000) A review on the genesis of coronal mass ejections. J Goophys Res 105:23,153-23,165. doi:10.1029/2000JA000005

Forbes TG, Priest ER (1995) Photospheric magnetic field evolution and eruptive flares. Astrophys J 446:377-389. doi:10.1086/175797

Gary GA (2001) Plasma beta above a solar active region: rethinking the paradigm. Sol Phys 203:71-86. doi:10.1023/A:1012722021820

Golub L, Deluca E, Austin G, et al. (2007) The X-ray telescope (XRT) for the Hinode mission. Sol Phys 243:63-86. doi:10.1007/s11207-007-0182-1

Green LM, Kliem B, Wallace A (2011) Photospheric flux cancellation and associated flux rope formation and eruption. Astron Astrophys 526:A2doi:10.1051/0004-6361/201015146

Guo Y, Ding MD, Cheng X, Zhao J, Pariat E (2013) Twist accumulation and topology structure of a solar magnetic flux rope. Astrophys J 779:157. doi:10.1088/0004-637X/779/2/157

Guo Y, Ding MD, Schmieder B (2010) Driving mechanism and onset condition of a confined eruption. Astrophys J Lett 725:L38-L42. doi:10.1088/2041-8205/725/1/L38

He H, Wang H (2008) Nonlinear force-free coronal magnetic field extrapolation scheme based on the direct boundary integral formulation. J Geophys Res Space Phys 113:A05S90. doi:10.1029/2007JA012441

Hirayama T (1974) Theoretical model of flares and prominences. I: Evaporating flare model. Sol Phys 34:323-338. doi:10.1007/BF00153671

Inoue S, Hayashi K, Magara T, Choe GS, Park YD (2014a) Magnetohydrodynamic simulation of the X2.2 solar flare on 2011 February 15. I. Comparison with the observations. Astrophys J 788:182. doi:10.1088/0004-637X/788/2/182

Inoue S, Hayashi K, Magara T, Choe GS, Park YD (2015) Magnetohydrodynamic simulation of the X2.2 solar flare on 2011 February 15. II. Dynamics connecting the solar flare and the coronal mass ejection. Astrophys 803:73. doi:10.1088/0004-637X/803/2/73

Inoue S, Hayashi K, Shiota D, Magara T, Choe GS (2013) Magnetic structure producing $\mathrm{X}$ - and M-class solar flares in solar active region 11158. Astrophys J 770:79. doi:10.1088/0004-637X/770/1/79

Inoue S, Kusano K (2006) Three-dimensional simulation study of flux rope dynamics in the solar corona. Astrophys J 645:742-756. doi:10.1086/503153

Inoue S, Kusano K, Magara T, Shiota D, Yamamoto $\Pi$ (2011) Twist and connectivity of magnetic field lines in the solar active region NOAA 10930. Astrophys J 738:161. doi:10.1088/0004-637X/738/2/161

Inoue S, Magara T, Pandey VS (2014b) Nonlinear force-free extrapolation of the coronal magnetic field based on the magnetohydrodynamic relaxation method. Astrophys J 780:101-. doi:10.1088/0004-637X/780/1/101

Inoue S, Magara T, Watari S, Choe GS (2012a) Nonlinear force-free modeling of a three-dimensional sigmoid observed on the sun. Astrophys J 747:65. doi:10.1088/0004-637X/747/1/65

Inoue S, Shiota D, Yamamoto TT (2012b) Buildup and release of magnetic twist during the X3.4 solar flare of 2006 December 13. Astrophys J 760:17. doi:10.1088/0004-637X/760/1/17

Isenberg PA, Forbes TG (2007) A three-dimensional line-tied magnetic field model for solar eruptions. Astrophys J 670:1453-1466. doi:10.1086/522025

Janvier M, Aulanier G, Bommier V (2014) Electric currents in flare ribbons: observations and three-dimensional standard model. Astrophys J 788:60. doi:10.1088/0004-637X/788/1/60 
Janvier M, Aulanier G, Pariat E, Démoulin P (2013) The standard flare model in three dimensions. III. Slip-running reconnection properties. Astron Astrophys 555:A77. doi:10.1051/0004-6361/201321164

Ji H, Wang H, Schmahl EJ, Moon YJ, Jiang Y (2003) Observations of the failed eruption of a filament. Astrophys J Lett 595:L135-L138

Jiang C, Feng X (2012) A new implementation of the magnetohydrodynamicsrelaxation method for nonlinear force-free field extrapolation in the solar corona. Astrophys J 749:135. doi:10.1088/0004-637X/749/2/135

Jiang C, Feng X (2013) Extrapolation of the solar coronal magnetic field from SDO/HMI magnetogram by a CESE-MHD-NLFFF. Code Astrophys J 769:144. doi:10.1088/0004-637X/769/2/144

Jiang C, Feng X, Wu ST, Hu Q (2013) Magnetohydrodynamic simulation of a sigmoid eruption of active region 11283. Astrophys J Lett 771:L30. doi:10.1088/2041-8205/771/2/L30

Jiang C, Wu ST, Feng X, Hu Q (2014a) Nonlinear force-free field extrapolation of a coronal magnetic flux rope supporting a large-scale solar filament from a photospheric vector magnetogram. Astrophys J Lett 786:L16. doi:10.1088/2041-8205/786/2/L16

Jiang C, Wu ST, Feng X, Hu Q (2014b) Formation and eruption of an active region sigmoid. I. A study by nonlinear force-free field modeling. Astrophys J 780:55. doi:10.1088/0004-637X/780/1/55

Karpen JT, Antiochos SK, DeVore CR (2012) The mechanisms for the onset and explosive eruption of coronal mass ejections and eruptive flares. Astrophys J 760:81. doi:10.1088/0004-637X/760/1/81

Kataoka R, Sato T, Kubo Y (2014) Radiation dose forecast of WASAVIES during ground-level enhancement. Space Weather 12:380-386. doi:10.1002/2014SW001053

Kliem B, Lin J, Forbes TG, Priest ER, Török T (2014) Catastrophe versus instability for the eruption of a toroidal solar magnetic flux rope. Astrophys J Let 789:46. doi:10.1088/0004-637X/789/1/46

Kliem B, Su YN, van Ballegooijen AA, DeLuca EE (2013) Magnetohydrodynamic modeling of the solar eruption on 2010 April 8. Astrophys J 779:129. doi:10.1088/0004-637X/779/2/129

Kliem B, Török T (2006) Torus instability. Phys Rev Lett 96:255002. doi:10.1103/PhysRevLett.96.255002

Kopp RA, Pneuman GW (1976) Magnetic reconnection in the corona and the loop prominence phenomenon. Sol Phys 50:85-98. doi:10.1007/BF00206193

Kosugi T, Matsuzaki K, Sakao T (2007) The Hinode (solar-B) mission: an overview. Sol Phys 243:3-17. doi:10.1007/s11207-007-9014-6

Kruskal MD, Kulsrud RM (1958) Equilibrium of a magnetically confined plasma in a toroid. Phis Fuluid 1:265-274. doi:10.1063/1.1705884

Kusano K, Bamba Y, Yamamoto TT (2012) Magnetic field structures triggering solar flares and coronal mass ejections. Astrophys J 760:31. 10.1088/0004-637X/760/1/31

Kusano K, Nishikawa K (1996) Bifurcation and stability of coronal magnetic arcades in a linear force-free field. Astrophys J 461:415-423. doi:10.1086/177070

Leake JE, Linton MG, Antiochos SK (2014) Simulations of emerging magnetic flux. II: The formation of unstable coronal flux ropes and the initiation of coronal mass ejections. Astrophys J 787:46. doi:10.1088/0004-637X/787/1/46

Lemen JR, Title AM, Akin DJ (2012) The atmospheric imaging assembly (AIA) on the solar dynamics observatory (SDO). Sol Phys 275:17. doi:10.1007/s11207-011-9776-8

Lin J, Forbes TG (2000) Effects of reconnection on the coronal mass ejection process. J Gophys Res 105:2375-2392. doi:10.1029/1999JA900477

Liu Y (2008) Magnetic field overlying solar eruption regions and kink and torus instabilities. Astrophys J 679:L151-. doi:10.1086/589282

Liu Y, Luhmann JG, Müller-Mellin R, et al. (2008) A comprehensive view of the 2006 December 13 CME: from the sun to interplanetary space. Astrophys J 689:563-571. doi:10.1086/592031

Louis RE, Kliem B, Ravindra B, Chintzoglou G (2015) Triggering an eruptive flare by emerging flux in a solar active-region complex. Solar Phys 290:3641-3662. doi:10.1007/s11207-015-0726-8

Low BC, Lou YQ (1990) Modeling solar force-free magnetic fields. Astrophys J 352:343-352. doi:10.1086/168541

Lynch BJ, Antiochos SK, DeVore CR, Luhmann JG, Zurbuchen TH (2008) Topological evolution of a fast magnetic breakout CME in three dimensions. Astrophys J 683:1192-1206. doi:10.1086/589738
Malanushenko A, Schrijver CJ, ML DeRosa, Wheatland MS (2014) Using coronal loops to reconstruct the magnetic field of an active region before and after a major flare. Astrophys J 783:102. doi:10.1088/0004-637X/783/2/102

Manchester WB, Gombosi TI, Roussev I (2004) Modeling a space weather event from the sun to the earth: CME generation and interplanetary propagation. J Geophys Res Space Phys 109:A02107. doi:10.1029/2003JA010150

Masuda S, Kosugi T, Hara H, Tsuneta S, Ogawara Y (1994) A loop-top hard X-ray source in a compact solar flare as evidence for magnetic reconnection. Nature 371:495-497. doi:10.1038/371495a0

McClymont AN, Mikic Z (1994) Thickness variations along coronal loops inferred from vector magnetograph data. Astrophys J 422:899-905. doi:10.1086/173781

McKenzie DE, Canfield RC (2008) Hinode XRT observations of a long-lasting coronal sigmoid. AandA 481:L65-L68. doi:10.1051/0004-6361: 20079035

Metcalf TR, De Rosa ML, Schrijver CJ, et al (2008) Nonlinear force-free modeling of coronal magnetic fields. II. Modeling a filament arcade and simulated chromospheric and photospheric vector fields. Sol Phys 247:269-299. doi:10.1007/s11207-007-9110-7

Miyoshi T, Kusano K (2011) A comparative study of divergence-cleaning techniques for multi-dimensional MHD schemes. Plasma Fusion Res 6:1124. doi:10.1585/pfr.6.2401124

Mikić Z, Barnes DC, Schnack DD (1988) Dynamical evolution of a solar coronal magnetic field arcade. Astrophys J 328:830-847. doi:10.1086/166341

Mikić Z, Lionello R, Mok Y, Linker JA, Winebarger AR (2013) The importance of geometric effects in coronal loop models. Astrophys J 773:94. doi:10.1088/0004-637X/773/2/94

Mikić Z, McClymont AN (1994) Deducing coronal magnetic fields from vector magnetograms. In solar active region evolution: comparing models with observations, ed. KS Balasubramaniam and GW Simon, 225-232. Astronomical Society of thePacific Conference Series, volume 68. Astronomical Society of the Pacific, San Francisco

Moore RL, Sterling AC, Hudson HS, Lemen JR (2001) Onset of the magnetic explosion in solar flares and coronal mass ejections. Astrophys J 552:833-848

Nakagawa Y (1974) Dynamics of the solar magnetic field. I. Method of examination of force-free magnetic fields. Astrophys J 190:437-440. doi:10.1086/152895

Nindos A, Patsourakos S, Vourlidas A, Tagikas C (2015) How common are hot magnetic flux ropes in the low solar corona? A statistical study of EUV observations. Astrophys J 808:117. doi:10.1088/0004-637X/808/2/117

Nishida K, Nishizuka N, Shibata K (2013) The role of a flux rope ejection in a three-dimensional magnetohydrodynamic simulation of a solar flare. Astrophys J Lett 775:L39. doi:10.1088/2041-8205/775/2/L39

Parenti S (2014) Solar prominences: observations. Liv Rev Sol Phys 11:1. doi:10.12942/Irsp-2014-1

Pesnell WD, Thompson BJ, Chamberlin PC (2012) The solar dynamics observatory (SDO). Sol Phys 275:3-15. doi:10.1007/s11207-011-9841-3

Peter H, Warnecke J, Chitta LP, Cameron RH (2015) Limitations of force-free magnetic field extrapolations: revisiting basic assumptions. Astron Astrophys 584:A68. doi:10.1051/0004-6361/201527057

Pevtsov AA, Berger MA, Nindos A, Norton AA, van Driel-Gesztelyi L (2014) Magnetic helicity, tilt, and twist. Space Sci Rev 186:285-324. doi:10.1007/s11214-014-0082-2

Priest E (2014) Magnetohydrodynamics of the sun. Cambridge University Press, UK

Priest ER, Forbes TG (1990) Magnetic field evolution during prominence eruptions and two-ribbon flares. Sol Phys 126:319-350. doi:10.1007/BF00153054

Priest ER, Forbes TG (2002) The magnetic nature of solar flares. Astron Astophys Rev 10:313-377. doi:10.1007/s001590100013

Régnier S, Amari T, Kersalé E (2002) 3D Coronal magnetic field from vector magnetograms: non-constant-alpha force-free configuration of the active region NOAA 8151. Astron Astrophys 392:1119-1127

Roumeliotis G (1996) The "stress-and-relax" method for reconstructing the coronal magnetic field from vector magnetograph data. Astrophys J 473:1095-1103. doi:10.1086/178219

Sakurai T (1981). Calculation of force-free magnetic field with non-constant $\alpha$ Sol Phys 69:343-359. doi:10.1007/BF00149999

Sakurai T (1982) Green's function methods for potential magnetic fields. Sol Phys 76:301-321. doi:10.1007/BF00170988 
Sakurai T (1989) Computational modeling of magnetic fields in solar active regions. Spa Sci Rev 51:11-48. doi:10.1007/BF00226267

Savcheva AS, van Ballegooijen AA, DeLuca EE (2012) Field topology analysis of a long-lasting coronal sigmoid. Astrophys J 744:78. doi:10.1088/0004-637X/744/1/78

Scherrer PH, Schou J, Bush Rl, et al. (2012) The helioseismic and magnetic imager (HMI) investigation for the solar dynamics observatory (SDO). Sol Phys 275:207-227. doi:10.1007/s11207-011-9834-2

Schmieder B, Aulanier G, Vršnak B (2015) Flare-C models: an observational perspective ME (invited review). Solar Phys 290:3457-3486. doi:10.1007/s11207-015-0712-1

Schrijver CJ, Aulanier G, Title AM, Pariat E, Delannée C (2011) The 2011 February 15 X2 flare, ribbons, coronal front, and mass ejection: interpreting the three-dimensional views from the solar dynamics observatory and STEREO guided by magnetohydrodynamic flux-rope modeling. Astrophys J 738:167. doi:10.1088/0004-637X/738/2/167

Schrijver CJ, Derosa ML, Metcalf TR, et al. (2006) Nonlinear force-free modeling of coronal magnetic fields part I: a quantitative comparison of methods. Sol Phys 235:161-190. doi:10.1007/s11207-006-0068-7

Schrijver CJ, Derosa ML, Metcalf T, et al. (2008) Nonlinear force-free field modeling of a solar active region around the time of a major flare and coronal mass ejection. Astrophys J 675:1637-1644. doi:10.1086/527413

Shibata K (1999) Evidence of magnetic reconnection in solar flares and a unified model of flares. Astrophys Space Sci 264:129-144

Shibata K, Magara T (2011) Solar flares: magnetohydrodynamic processes. Liv Rev Sol Phys 8:6. doi:10.12942/Irsp-2011-6

Shiota D, Isobe H, Chen PF, Yamamoto TT, Sakajiri T, Shibata K (2005) Self-consistent magnetohydrodynamic modeling of a coronal mass ejection, coronal dimming, and a giant cusp-shaped arcade formation. Astrophys J 634:663-678. doi:10.1086/496943

Shiota D, Kusano K, Miyoshi T, Shibata K (2010) Magnetohydrodynamic modeling for a formation process of coronal mass ejections: interaction between an ejecting flux rope and an ambient field. Astrophys J 718:1305-1314. doi:10.1088/0004-637X/718/2/1305

Shiota D, Tsuneta S, Ito H, Kusao K, Nishikawa N, Suzuki TK (2012) Fine structure in three dimensional magnetic field in polar region. Hinode-3: The 3rd Hinode Science Meeting, Proceedings of the conference held 1-4 December 2009 at Hitotsubashi Memorial Hall, ASP Conference Series, Vol. 454, 375-378. Astronomical Society of the Pacific, San Francisco

Sterling AC, Hudson HS (1997) Yohkoh SXT observations of X-ray "dimming" associated with a halo coronal mass ejection. Astrophys J Lett 491:L55-L58. doi:10.1086/311043

Sturrock PA (1966) Model of the high-energy phase of solar flares. Nature 211:695-700. doi:10.1038/211695a0

Su Y, Golub L, van Ballegooijen A, et al. (2007) Evolution of the sheared magnetic fields of two X-class flares observed by Hinode/XRT. Pub Astron Sci Japan 59:785-791. doi:10.1093/pasj/59.sp3.S785

Su Y, Surges V, van Ballegooijen A, DeLuca E, Golub L (2011) Observations and magnetic field modeling of the flare/coronal mass ejection event on 2010 April 8. Astrophys J 734:53. doi:10.1088/0004-637X/734/1/53

Su Y, van Ballegooijen A, Lites BW, Deluca EE, Golub L, Grigis PC, Huang G, Ji H (2009) Observations and nonlinear force-free field modeling of active region 10953. Astrophys J 691:105. doi:10.1088/0004-637X/691/1/105

Sun X, Hoeksema JT, Liu Y, Wiegelmann T, Jayashi K, Chen Q, Thalmann J (2012) Evolution of magnetic field and energy in a major eruptive active region based on SDO/HMI observation. Astrophys J 748:77. doi:10.1088/0004-637X/748/2/77

Tanaka T (1995) Generation mechanisms for magnetosphere-ionosphere current systems deduced from a three-dimensional MHD simulation of the solar wind-magnetosphere-ionosphere coupling processes. J Geophys 100:12,057-12,074. doi:10.1029/95JA00419

Thalmann JK, Inhester B, Wiegelmann T (2011) Estimating the relative helicity of coronal magnetic fields. Sol Phys 272:243-255. doi:10.1007/s11207-011-9826-2

Titov VS, Démoulin P (1999) Basic topology of twisted magnetic configurations in solar flares. Astrophys Astron 351:707-720

Tiwari SK, Alexander CE, Winebarger AR, Moore RL (2014) Trigger mechanism of solar subflares in a braided coronal magnetic structure. Astrophys J Let 795:L24. doi:10.1088/2041-8205/795/1/L24

Toriumi S, lida Y, Bamba Y, Kusano K, Imada S, Inoue S (2013) The magnetic systems triggering the M6.6 class solar flare in NOAA active region 11158 Astrophys J 773:128. doi:10.1088/0004-637X/773/2/128
Török T, Kliem B, Titov VS (2004) Ideal kink instability of a magnetic loop equilibrium. Astron Astrophys 413:L27-L30. doi:10.1051/0004-6361:20031691

Török T, Kliem B (2005) Confined and ejective eruptions of kink-unstable flux ropes. Astrophys J Lett 630:L97-L100. doi:10.1086/462412

Török T, Kliem B (2007) Numerical simulations of fast and slow coronal mass ejections. Astron Nach 328:743-746. doi:10.1002/asna.200710795

Török T, Aulanier G, Schmieder B, Reeves KK, Golub L (2009) Fan-spine topology formation through two-step reconnection driven by twisted flux emergence. Astrophys J 704:485-495. doi:10.1088/0004-637X/704/1/485

Török T, Berger MA, Kliem B (2010) The writhe of helical structures in the solar corona. Astron Astrophys 516:A49. doi:10.1051/0004-6361/200913578

Török T, Panasenco O, Titov VS, Mikić Z, Reeves KK, Velli M, Linker JA, De Toma $\mathrm{G}$ (2011) A model for magnetically coupled sympathetic eruptions. Astrophys J Lett 739:L63. doi:10.1088/2041-8205/739/2/L63

Tóth $\mathrm{G}$ (2000) The $\nabla \cdot \boldsymbol{B}=0$ constraint in shock-capturing magnetohydrodynamics codes. J Comput Phys 161:605-652. doi:10.1006/jcph.2000.6519

Tóth G, Sokolov IV, Gombosi TI, et al (2005) Space weather modeling framework: a new tool for the space science community. J Geophys Res 110:A12226. doi:0.1029/2005JA011126

Tsuneta S (1996) Structure and dynamics of magnetic reconnection in a solar flare. Astrophys J 456:840-849. doi:10.1086/176701

Tsuneta S, Hara H, Shimizu T, et al. (1992) Observation of a solar flare at the limb with the $\mathrm{YOHKOH}$ soft X-ray telescope. Pub Astron Soc Japan 44:L63-L69

Tsuneta S, Ichimoto K, Katsukawa Y, et al. (2008) The solar optical telescope for the Hinode mission: an overview. Sol Phys 249:167-169. doi:10.1007/s11207-008-9174-z

Valori G, Démoulin P, Pariat E (2012) Comparing values of the relative magnetic helicity in finite volumes. Sol Phys 278:347-366

Valori G, Kliem B, Fuhrmann M (2007) Magnetofrictional extrapolations of Low and Lou's force-free equilibria. Sol Phys 245:263-285

Valori G, Kliem B, Keppens R (2005). Extrapolation of a nonlinear force-free field containing a highly twisted magnetic loop Astrophsys Astron 433:335-347. doi:10.1051/0004-6361:20042008

van Ballegooijen AA (2004) Observations and modeling of a filament on the sun. Astrophys J 612:519-529. doi:10.1086/422512

van Ballegooijen AA, PMartens CH (1989) Formation and eruption of solar prominences. Astrophys J 343:971-984. doi:10.1086/167766

van Ballegooijen AA, Priest ER, Mackay DH (2000) Mean field model for the formation of filament channels on the Sun. Astrophys J 539:983-994. doi:10.1086/309265

van Driel-Gesztelyi L, Baker D, Török T, et al. (2014) Coronal magnetic reconnection driven by CME expansion: the 2011 June 7 event. Astrophys J 788:85. doi:10.1088/0004-637X/788/1/85

Vemareddy P, Ambastha A, Wiegelmann T (2013) Magnetic structure of solar active region NOAA 11158. Bull Astron Soc India 41:183

Vemareddy P, Zhang J (2014) Initiation and eruption process of magnetic flux rope from solar active region NOAA 11719 to earth-directed CME. Astrophys J 797:80. doi:10.1088/0004-637X/797/2/80

Wang H, Liu C (2015) Structure and evolution of magnetic fields associated with solar eruptions. Res Astron Astrophys 15:145-174. doi:10.1088/1674-4527/15/2/001

Wang S, Liu C, Liu R, Deng N, Liu Y, Wang H (2012) Response of the photospheric magnetic field to the X2.2 flare on 2011 February 15. Astrophys J Lett 745:L17. doi:10.1088/2041-8205/745/2/L17

Wang R, Yan Y, Tan B (2013) Three-dimensional nonlinear force-free field reconstruction of solar active region 11158 by direct boundary integral equation. Sol Phys 288:507-529. doi:10.1007/s11207-013-0422-5

Wheatland MS, Leka KD (2011) Achieving self-consistent nonlinear force-free modeling of solar active regions. Astrophsys J 728:112. doi:10.1088/0004-637X/728/2/112

Wheatland MS, Régnier S (2009) A self-consistent nonlinear force-free solution for a solar active region magnetic field. Astrophys J Lett 700:L88-L91. doi:10.1088/0004-637X/700/2/L88

Wheatland MS, Sturrock PA, Roumeliotis G (2000) An optimization approach to reconstructing force-free fields. Astrophys J 540:1150-1155. doi:10.1086/309355

Wiegelmann T (2004) Optimization code with weighting function for the reconstruction of coronal magnetic fields. Sol Phys 219:87-108. doi:10.1023/B:SOLA.0000021799.39465.36 
Wiegelmann T, Sakurai T (2012) Solar force-free magnetic fields. Liv Rev Sol Phys 9:5. doi:10.12942/lrsp-2012-5

Wu ST, Sun MT, Chang HM, Hagyard MJ, Gary GA (1990) On the numerical computation of nonlinear force-free magnetic fields. Astrophys J 362:698-708. doi:10.1086/169307

Yan Y (1995) The 3-D boundary element formulation of linear force-free magnetic fields with finite energy content in semi-infinite space. Sol Phys 159:97-113. doi:10.1007/BF00733034

Yan Y, Sakurai T (2000) New boundary integral equation representation for finite energy force-free magnetic fields in open space above the Sun. Sol Phys 195:89-109. doi:10.1023/A:1005248128673

Yang Y-H, Chen PF, Hsieh M-S, et al. (2014) Characteristics of the photospheric magnetic field associated with solar flare initiation. Astrophys J 786:72. doi:10.1088/0004-637X/786/1/72

Yokoyama T, Akita K, Morimoto T, Inoue K, Newmark J (2001) Clear evidence of reconnection inflow of a solar flare. Astrophys J Lett 546:L69-L72. doi:10.1086/318053

Yokoyama T, Shibata K (1994) What is the condition for fast magnetic reconnection? Astrphys J Lett 436:L197-L200. doi:10.1086/187666

Yokoyama T, Shibata K (2001) Magnetohydrodynamic simulation of a solar flare with chromospheric evaporation effect based on the magnetic reconnection model. Astrophys J 549:1 160-1174. doi:10.1086/319440

Zhao J, Li H, Pariat E, Schmieder B, Guo Y, Weigelmann T (2014) Temporal evolution of the magnetic topology of the NOAA active region 11158. Astrophys J 787:88. doi:10.1088/0004-637X/787/1/88

Zuccarello FP, Meliani Z, Poedts S (2012) Numerical modeling of the initiation of coronal mass ejections in active region NOAA 9415. Astrophys J 758:117. doi:10.1088/0004-637X/758/2/117

\section{Submit your manuscript to a SpringerOpen ${ }^{\circ}$ journal and benefit from:}

- Convenient online submission

- Rigorous peer review

- Immediate publication on acceptance

- Open access: articles freely available online

- High visibility within the field

- Retaining the copyright to your article

Submit your next manuscript at $\gg$ springeropen.com 